SU-ITP-04-07

UCB-PTH-04/05

LBNL-54620

hep-th/0403198

\title{
Comments on Cosmic Censorship in AdS/CFT
}

\author{
Veronika E. Hubeny ${ }^{a}$, Xiao Liu ${ }^{a, b}$, Mukund Rangamani $^{c, d}$, And Stephen Shenker ${ }^{a}$ \\ ${ }^{a}$ Department of Physics, Stanford University, Stanford, CA 94305, USA \\ ${ }^{b}$ SLAC, Stanford University, Stanford, CA 94309, USA \\ ${ }^{c}$ Department of Physics, University of California, Berkeley, CA 94720, USA \\ ${ }^{d}$ Theoretical Physics Group, LBNL, Berkeley, CA 94720, USA
}

\begin{abstract}
Recently Hertog, Horowitz, and Maeda (HHM) (hep-th/0310054) have proposed that cosmic censorship can be violated in the AdS/CFT context. They argue that for certain initial data there is insufficient energy available to make a black hole whose horizon is big enough to cloak the singularity that forms. We have investigated this proposal in the models HHM discuss and have thus far been unable to find initial data that provably satisfy this criterion, despite our development of an improved lower bound on the size of the singular region. This is consistent with recent numerical results (hep-th/0402109). For certain initial data, the energies of our configurations are not far above the lower bound on the requisite black hole mass, and so it is possible that in the exact time development naked singularities do form. We go on to argue that the finite radius cut-off $\mathrm{AdS}_{5}$ situation discussed by HHM displays instabilities when the full 10D theory is considered. We propose an $\mathrm{AdS}_{3}$ example that may well be free of this instability.
\end{abstract}

March 2004

\footnotetext{
veronika@itp.stanford.edu, liuxiao@itp.stanford.edu, mukund@socrates.berkeley.edu, sshenker@stanford.edu
} 


\section{Introduction}

Curvature singularities are a rather generic feature in general relativity. As demonstrated by the singularity theorems [1], even relatively mild generic initial data can evolve to a singularity. Indeed, in nature, we expect singularities inside black holes as well as cosmological ones. Of course, the laws of general relativity break down at a singularity, thereby precluding us from evolving the classical spacetime into its causal future. The feeling that the collapse to a singularity in one region of spacetime should not terminate the classical evolution in regions arbitrarily far away prompted Penrose [2] to conjecture that these singularities are generically cloaked by event horizons, and therefore hidden inside black holes. In other words, there is some cosmic censor, which prevents the formation of naked singularities (i.e., those visible to distant observers). More specifically, the Cosmic Censorship Conjecture states that smooth and generic initial data with "reasonable" matter cannot evolve to a naked singularityt.

The Cosmic Censorship Conjecture, first formulated over three decades ago, has enjoyed a vibrant history (for a recent review of the subject, see e.g. [3]) and remains one of the most important and intriguing questions in general relativity today. While no proof has yet been found, cosmic censorship is often assumed to hold. On the other hand, numerous "counter-examples" have been proposed; typically this has led to tighter formulations of the conjecture. For example, naked singularities can be produced with fine-tuned initial conditions [4], with pressureless matter [5], etc., whereas various proposals with generic and reasonable initial conditions, such as overcharging a black hole [6], are too delicate to prove the production of naked singularities. Recently Hertog, Horowitz, and Maeda [7] have argued that cosmic censorship can be violated for configurations with a scalar field coupled to gravity in asymptotically AdS spacetimes. This would give one of the first examples of cosmic censorship violation using generic initial conditions with reasonable matter目.

The AdS boundary conditions facilitate formation of naked singularities, as we will review below. More intriguingly, however, they are of importance in string theory: Ac-

1 In fact there are two versions: the weak cosmic censorship conjecture precludes the singularities to be visible asymptotically; while the strong cosmic censorship conjecture disallows formation of timelike singularities, even if they are inside black holes. Here we shall consider only the weak version.

2 See [8] for cosmic censorship violation in an asymptotically dS case. 
cording to the well-known AdS/CFT correspondence [9, 10,11, 12], Type IIB string theory on asymptotically $\mathrm{AdS}_{5} \times \mathbf{S}^{5}$ spacetime is dual to a 4 -dimensional $\mathcal{N}=4$ Super YangMills gauge theory "living on the boundary" of AdS. Since the correspondence holds at the quantum level, we expect that any naked singularity produced in the bulk of AdS will be resolved by the gauge theory. After all, the CFT evolution is well-defined and should not terminate at some finite time when the gauge theory comes into causal contact with the singularity. So embedding naked singularities in AdS/CFT would provide a robust way to study quantum gravity in these extreme environments. The current methods [13] for studying spacelike singularities in AdS/CFT focus on the black hole singularity which lives behind a horizon, and so requires delicate techniques to investigate.

As a first step to the program of using the dual field theory description to see how singularities are resolved in a quantum theory of gravity, one needs to construct simple configurations in string theory which evolve to a naked singularity.

Unfortunately, the results of [7] cannot be directly applied to this endeavor, because the action used there is not one derived from string theory. A suitable potential for the scalar field is chosen by hand rather than deriving it from the appropriate supergravity action. However, in a very interesting follow-up paper [14], Hertog, Horowitz, and Maeda (HHM) do examine cosmic censorship in the AdS/CFT context. In particular, they argue that naked singularities will be produced generically even in $5 \mathrm{D} \mathcal{N}=8$ supergravity, a consistent truncation of the low energy limit of string theory on $\operatorname{AdS}_{5} \times \mathbf{S}^{5}$. The essence of their argument is to produce a simple configuration that reliably evolves into a singularity, and then show that there is insufficient energy available to make a black hole whose horizon is big enough to cloak the singularity. Recently, this claim has been challenged in [15], whose numerical study of the HHM process suggests 3 formation of black holes rather than naked singularities.

In this note, we study the parameter space for initial data with the purpose of identifying a region where one can reliably argue that naked singularities form and moreover the dual CFT is well-behaved. In the next section, we give a brief review of the two HHM papers. In Section 3 we discuss the infinite radius non cut-off AdS/CFT context, consis-

3 We should point out, though, that this numerical study only indicates the presence of trapped surfaces. While this is suggestive, a compelling demonstration might well require evolving the initial data far enough into the future to see the exterior metric settle into the Schwarzschild-AdS black hole geometry. 
tently truncated to 5D supergravity, studied by HHM. We are unable to find any initial data where we can rigorously show that the configuration possesses too little energy to make a black hole of the requisite size. The energy excess is small in certain parameter regimes and so the possibility exists that a more accurate (and hence larger) estimate of the size of the singular region will show that a naked singularity must form.

In Section 4 we turn to the finite radius cut-off AdS version introduced in [14](v2). The situation is similar to the infinite radius situation. In addition, here we show that the full 10D theory is unstable to D3-branes moving to the boundary, using both bulk and boundary CFT arguments. The true late time behavior of the theory will be dominated by this instability and so predictions for the full theory from the five dimensional analysis are problematic.

In Section 5, we focus on a special case of cut-off 3-dimensional AdS, where the instability seems absent. There may well be other strong 10 dimensional dynamics present in this system though. Again we do not find any initial data that reliably produce naked singularities.

We end with a summary and some discussion in Section 6. Most of the detailed calculations and numerical estimates are relegated to Appendices, where among other things we refine and extend the estimates of HHM to a more general set-up in $d$ dimensions.

\section{Review of HHM}

In [7], Hertog, Horowitz, and Maeda consider 4-dimensional gravity coupled to a scalar field $\phi$ with the potential $V(\phi)$. The potential, sketched in Fig.1a, has two negative minima, a global one at $\phi=0$ and a local one at $\phi_{1}$; and the solutions are chosen so as to asymptotically approach $\phi_{1}$ (i.e., $V\left(\phi_{1}\right)$ determines the AdS radius of the resulting asymptotically AdS spacetime). As a physical requirement, the potential $V(\phi)$ is chosen so as to satisfy the positive energy theorem for all solutions with these boundary conditions. More specifically, the (time-symmetric and spherically symmetric) initial conditions consist of the field profile as sketched in Fig.1b: the field is near the global minimum $\phi \approx 0$ in some interior region $r \leq R_{0}$, and then smoothly asymptotes to the local minimum $\phi_{1}$.

In the central region, where the field is homogeneous and isotropic, the evolution is determined by the standard FRW equations. In particular, since a homogeneous scalar field rolling down a potential to a negative minimum becomes singular, our spacetime will develop a spacelike singularity in the domain of dependence of the central region. Will this 

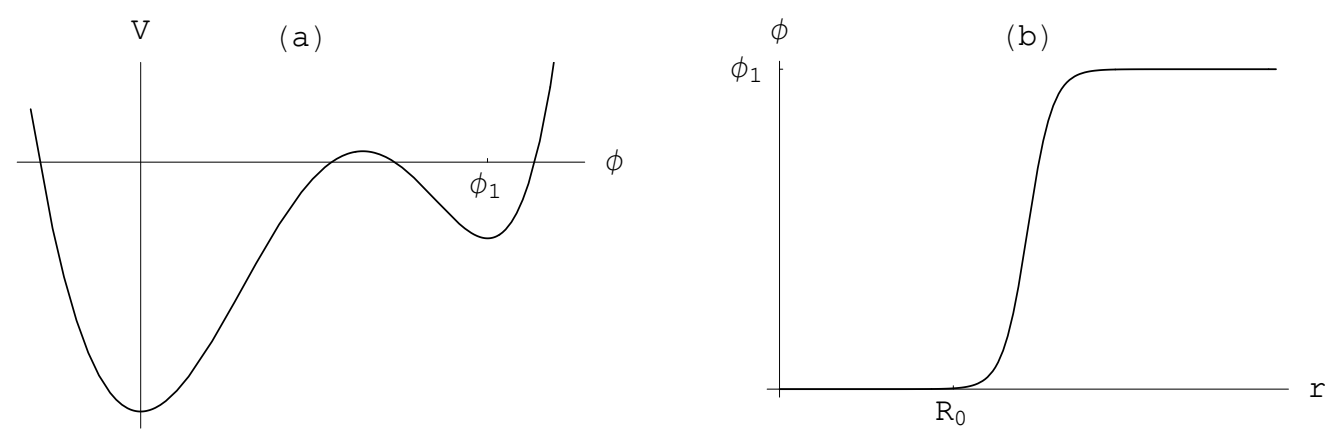

Fig. 1: Potential $V(\phi)$ and the field profile $\phi(r)$ used by HHM [7] in the purely gravitational context.

singularity be cloaked by an event horizon, or can it be naked? In order for the singularity to be hidden inside a black hole, the black hole horizon has to be large enough to encompass the singularity, so correspondingly the black hole mass has to be large enough. On the other hand, by mass conservation, if the initial configuration forms a black hole, the black hole mass can be no larger than the initial mass. Thus, a naked singularity must result if the mass $M_{\text {config }}$ of the initial configuration is smaller than the mass $M_{\mathrm{BH}}$ of the smallest black hole large enough to cloak the ensuing singularity.

By cleverly tuning the potential, the authors of [7] show that there exists an open set of initial conditions which satisfy this criterion. In particular, by making the interior region very large, $R_{0} \gg 1$, the black hole size must correspondingly scale as $R_{0}^{3}$. Although generically the mass of the initial configuration also scales as $R_{0}^{3}$, by tuning the potential, they are able to cancel this leading contribution, so that instead the mass scales only as $R_{0}$. Hence, by taking $R_{0}$ large enough, it appears that they can easily satisfy the naked singularity condition, $M_{\text {config }}<M_{\mathrm{BH}}$. will explain in Appendix B.6. This same issue is also discussed in [16]. Evidence pointing toward this conclusion was presented in [17]. 目

4 The fact that $M_{\mathrm{BH}} \sim r_{+}^{3}$ for large horizon radii $r_{+}$is due to the AdS boundary conditions; to form a black hole of a given size, more mass is required in AdS than in flat space. Since in the latter $M_{\mathrm{BH}} \sim r_{+}$, more detailed study is necessary to certify whether a similar mechanism would allow the formation of a naked singularity in an asymptotically flat spacetime.

5 Recently the authors of [18] have argued that there is a loophole in this argument: the singularity need not be cloaked by a black hole which asymptotically looks large and static, but instead the field may act as a forever expanding domain wall of negative energy, leaving room inside for a large black hole. This can’t happen in a system satisfying the positive energy theorem, 
To consider the analogous situation in the AdS/CFT context, one no longer has the freedom to pick an arbitrary potential, which makes the arguments for cosmic censorship violation much more involved. Nevertheless, HHM [14] argue that naked singularities can still be formed generically. This relies on constructing configurations which, as in the previous case, collapse to a singularity, but from the bulk point of view initially have negative energy, and therefore seemingly cannot form a black hole.

In particular, HHM use the well-known fact that in AdS, scalar fields can have negative mass squared, but nevertheless can be stable, provided they satisfy the BreitenlohnerFreedman $(\mathrm{BF})$ bound $m^{2} \geq m_{\mathrm{BF}}^{2}=-\frac{(d-1)^{2}}{4}$ in $\mathrm{AdS}_{d}$ [19,20]. While the positive energy theorem is necessarily satisfied for $m^{2}>m_{\mathrm{BF}}^{2}$, for fields saturating the BF bound $m^{2}=$ $m_{\mathrm{BF}}^{2}$, the total bulk (ADM) energy (which is normalized such that pure AdS has zero energy) can be arbitrarily negative [14]. In the bulk, this "bulk energy" is what governs the evolution in the domain of dependence of the initial data surface 6 . However, the dual CFT energy corresponds to the bulk energy plus a finite counter-term, which may be viewed as the boundary contribution. This boundary term vanishes when the field falls off faster than the fall-off given by the BF bound, but it is present for the BF-saturating fields. This ensures that the CFT energy is positive and conserved.

HHM concentrate on the $\mathcal{N}=8$ gauged supergravity on $\mathrm{AdS}_{5}$, which arises as the low energy limit of string theory with $\mathrm{AdS}_{5} \times \mathbf{S}^{5}$ boundary conditions, and is conjectured to be a consistent 5 dimensional truncation of 10 dimensional Type IIB supergravity [21].

In global coordinates, with $\mathrm{AdS}_{5}$ given by

$$
d s^{2}=-\left(1+r^{2}\right) d t^{2}+\frac{d r^{2}}{1+r^{2}}+r^{2} d \Omega_{3}^{2}
$$

the solutions to the scalar wave equation $\left(\nabla^{2}-m^{2}\right) \phi=0$ fall off asymptotically as $\phi(r) \sim$ $r^{1 / \lambda_{ \pm}}$where $\lambda_{ \pm} \equiv 2 \pm \sqrt{4+m^{2}}$. When $m^{2}=m_{\mathrm{BF}}^{2}=-4$, the solutions behave as $1 / r^{2}$ and $\log r / r^{2}$. Since the latter is non-normalizable (unless one considers the cut-off version of the correspondence as discussed in Section 4), HHM focus on the former. From the higher dimensional point of view, the BF-saturating scalars correspond to the $\ell=2$ modes on

so we do not consider it further. We thank Gary Horowitz for pointing this out to us; $c f$. 16.

6 More specifically, as explained in Appendix A, if we write the spatial metric on the initial slice as in $(\mathrm{A} .2), d s_{\Sigma}^{2}=\left(1-\frac{m(r)}{3 \pi^{2} r^{2}}+r^{2}\right)^{-1} d r^{2}+r^{2} d \Omega_{3}^{2}$, then the ADM mass is given simply by $\lim _{r \rightarrow \infty} m(r)$; and the bulk evolution is sensitive to the local metric, i.e., to the function $m(r)$. The generalization to $d$ dimensions can be found in (A.18). 

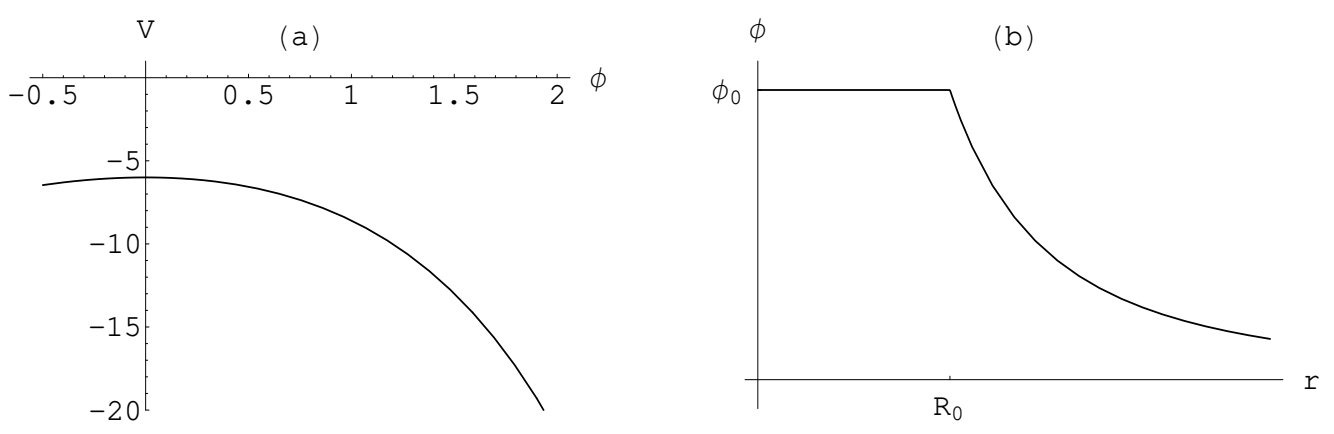

Fig. 2: Supergravity potential $V(\phi)$ and the field profile $\phi(r)$ used by HHM [14] in the AdS/CFT context.

the $\mathbf{S}^{5}$ 22]. There is one special field which does not source any of the others, so HHM set all other fields to zero and concentrate on this single scalar field, $\phi$. The action (with AdS radius set to 1 ) then reduces to

$$
S=\int \sqrt{-g}\left[\frac{1}{2} R-\frac{1}{2}(\nabla \phi)^{2}-V(\phi)\right]
$$

where the potential $V(\phi)$, plotted in Fig.2a, is given by

$$
V(\phi)=-2 e^{2 \phi / \sqrt{3}}-4 e^{-\phi / \sqrt{3}}
$$

The particular field configuration that HHM consider as initial conditions (which they again take to be time-symmetric and spherically symmetric) is plotted in Fig.2b, and is given by

$$
\phi(r)=\phi_{0} \equiv \frac{A}{R_{0}^{2}} \quad r \leq R_{0}, \quad \phi(r)=\frac{A}{r^{2}} \quad r>R_{0} .
$$

In the domain of dependence of the internal region $r \leq R_{0}$ the field evolves according to the FRW equations to a spacelike singularity. The detailed evolution is described in Appendix B for $d$ dimensions; the HHM set-up corresponds to $d=5$. Physically, the field in the central region rolls down the potential and diverges. However, since the asymptotic field is pinned to the top of the potential, the spacetime is asymptotically AdS.

The main question concerns the evolution of the field outside the domain of dependence of the internal region. There are several a-priori possibilities as to what can happen: 1) The spacelike singularity joins on to the spacelike black hole singularity, i.e., the field configuration collapses to a black hole, and the CFT doesn't "see" the singularity at 
any finite timel. 2) The singularity stops inside the bulk or becomes timelike (these are indistinguishable from the point of view of the classical evolution, since the evolution in the future domain of influence of the singularity is not well-defined). The CFT then "sees" this naked singularity at some finite time (presumably, there are local observables which reflect the causal contact with the high curvature region), but since the evolution in the CFT is well defined, ultimately this should "resolve" the naked singularity. This is the case that HHM argue for. 3) The singularity continues and eventually "hits" the boundary at a finite CFT time-a Big Crunch. This is the most drastic (and therefore presumably least likely) outcome, for then the CFT would seem to end or change abruptly. Remarkably, Dafermos [23] has proven that in the case of scalar fields with potentials that are bounded from below (as in the example of [7]) if cosmic censorship is violated, then a Big Crunch must necessarily occur.

\section{AdS/CFT without a cut-off}

To prove the formation of naked singularities, HHM first argued that the initial configuration (which is guaranteed to evolve to a singularity) does not have sufficient energy to form a black hole. In particular, the ADM energy of the initial configuration is negative. And although the CFT energy is guaranteed to be positive, it is the bulk energy (given by the bulk metric) which determines the bulk evolution. Unfortunately, this simple kinematic argument is not easily applicable for BF-saturating fields and HHM have already pointed out a possible loophole in this argument [14](v2). Essentially, as we explain below, the bulk energy rises too fast, and the conserved energy is too large.

The ADM bulk energy is not conserved because of the slow fall off of $\phi$. Only the CFT energy, which includes the boundary term is conserved. Consequently, outside the domain of dependence of the initial data the bulk energy could in principle get as large as the CFT energy. One may think of this in terms of the extra energy stored "at the boundary" coming into the bulk. Fig.3 illustrates the following argument that the bulk energy can in principle change on timescales shorter than that necessary for the "formation" of the black hole.

As reviewed in Appendix B, the singularity forms on a (part of) constant FRW time $T$ slice. However, the singularity is guaranteed to form only in the domain of dependence

7 There may of course still be signatures of the black hole singularity, as discussed in [13], but these do not correspond to local observables on the boundary. 


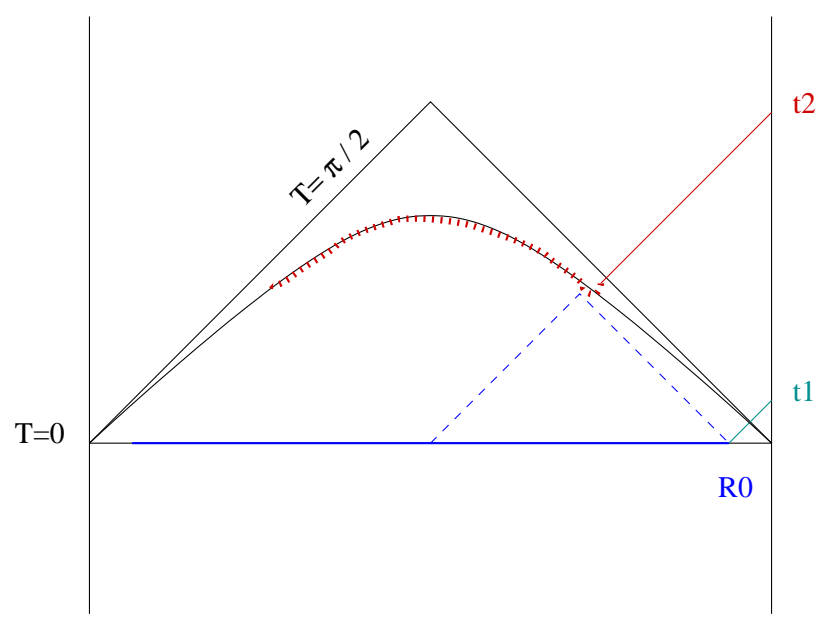

Fig. 3: Causal diagram of the formation of the singularity for small initial fields $\phi_{0}$.

of the inner region, which requires $R_{0}$ to be quite large. This in turn implies that the propagation of the "kink" in the initial field profile (2.4) (i.e. the domain of influence of the inner region) reaches the boundary at fairly early times (denoted $t_{1}$ in the diagram). So at $t_{1}$, we would expect the bulk energy to start changing. Indeed, numerical evolution [15] of the data (2.4) shows that the bulk energy starts to increase on short timescales, rising quickly to the CFT energy value.

We cannot easily determine the behavior of the singularity outside the domain of dependence of the inner $\left(r<R_{0}\right)$ region; the singularity could in principle turn around to become almost null (or even timelike). But that means that to have a black hole, with everywhere spacelike singularity, we only need to "collapse" it much later; in pure AdS this would be at time $t_{2} \gg t_{1}$ as sketched. Conversely, in order to be certain that a naked singularity will be produced, one would have to argue that the ADM energy doesn't change (or at least become too large) by time $t_{2}$. Actually, the situation is even worse, since if the black hole did form, and on the causal diagram the singularity reached the boundary at $t_{2}$, then from the boundary point of view $t_{2}$ would really be infinite.

To say this another way, given that there is enough CFT energy to make the required black hole, to motivate naked singularity we would have to show that the black hole forms much "later" than the singularity. Qualitatively, we expect the characteristic time for singularity formation (from weak initial fields) should be of order the AdS time. The time for black hole formation should be of a similar order. So there is no parametric separation in time scales that would guarantee singularity formation well before black 
hole formation. For very large energy initial conditions, like (2.4) at large $\phi_{0}$, we expect both time scales to shorten in a way which is unfavorable for the formation of naked singularities. In particular, for strong initial fields and large inner region the CFT time to see a naked singularity would be $\sim \frac{1}{R_{0}}$. On the other hand, if a large black hole did form, we would expect it to settle down (and the CFT to thermalize) on timescales given by the quasinormal mode frequencies, which scale with the black hole temperature [24], and therefore are given by $\mathrm{O} \sim \frac{1}{R_{0} \phi_{0}^{1 / 2}} \ll \frac{1}{R_{0}}$. Although the quasinormal modes represent the late-time behavior of the black hole, given the parametric separation in the time scales, this argument suggests that even if the singularity becomes locally timelike, it would still be cloaked by the horizon. As we shall see, there do exist large $\phi_{0}$ configurations of bounded energy. For these configurations we expect all time scales to be $\sim 1 / R_{0}$.

We have seen that the bulk energy (which is initially too small to form a black hole) does not necessarily remain small long enough to forbid black hole formation. The kinematic argument for naked singularities would still apply, though, if the conserved CFT energy were too small to make a sufficiently large black hold 9 . But, as we describe below, this energy appears sufficient to create the required black hole. Here we review this result in a regulated context 10 .

To understand this issue better, it is helpful to envision a sequence of initial data $\phi_{\epsilon}(r)$ that approach the configuration (2.4) as $\epsilon \rightarrow 0$ and differ significantly from it only at larger and larger $r$. Explicitly, take

$$
\phi_{\epsilon}(r)=\phi_{0} \equiv \frac{A}{R_{0}^{2+\epsilon}} \quad r \leq R_{0}, \quad \phi_{\epsilon}(r)=\frac{A}{r^{2+\epsilon}} \quad r>R_{0} .
$$

For finite $\epsilon$, the fall off at large $r$ is fast enough that the bulk ADM energy coincides with the CFT energy and is conserved. We can imagine embedding these configurations in the boundary CFT with an effective UV CFT cutoff. Field theory intuition tells us that any physically meaningful phenomenon in the boundary CFT should be understandable as the

8 As we show later, the total energy in the configuration (2.4) is approximately $R_{0}^{4} \phi_{0}^{2}$. If a black hole forms, as is kinematically favorable, its mass is likely to be the full mass of the configuration, so that its horizon would be roughly $r_{+} \sim R_{0} \phi_{0}^{1 / 2}$. Since this is a very large black hole, the corresponding black hole temperature would scale also as $T \sim R_{0} \phi_{0}^{1 / 2}$.

9 On general grounds we expect the CFT to thermalize eventually, resulting in a large black hole in the bulk. So we expect any naked singularity to eventually retreat behind the black hole horizon.

10 The following argument was motivated by a discussion with Juan Maldacena. 
limit of the behavior of a sequence of UV cut-off CFTs. So if a naked singularity is formed it should form for finite but small $\epsilon$ and continue smoothly to $\epsilon=0$.

The ADM (equal to CFT) energy of (3.1) can be evaluated by using the constraint equations (A.1). The detailed calculation is outlined in Appendix A. As explained there, the $\epsilon \rightarrow 0$ limit reveals a manifestly positive answer for the ADM mass. We quote here the dominant piece for small $\phi_{0}$;

$$
M_{\text {config }}=\pi^{2} \phi_{0}^{2} R_{0}^{4}+\cdots
$$

Since this energy is conserved and represents the largest possible value for the bulk energy (because the boundary term is explicitly positive), we can now ask whether this energy suffices to form a black hole. In $\mathrm{AdS}_{5}$ a Schwarzschild black hole with horizon size $r_{+}$(in AdS units) has mass

$$
M_{B H}=3 \pi^{2} r_{+}^{2}\left(r_{+}^{2}+1\right)
$$

We need to know the minimal Schwarzschild radius $R_{s}$ that will enclose the singularity. (By the area theorem, the size of the event horizon of a black hole cannot shrink, so the final black hole can only get bigger: $r_{+} \geq R_{s}$.) A simple ray tracing argument as in [14], reveals 11 that one needs a Schwarzschild radius

$$
R_{s}>R_{0} \phi_{0}^{2 / 3}, \quad \text { for } \quad \phi_{0} \ll 1
$$

Evaluating at the lower bound,

$$
M_{\mathrm{BH}}>3 \pi^{2} \phi_{0}^{8 / 3} R_{0}^{4}
$$

where we have restricted ourselves to the most favorable region, $R_{s} \gg 1$.

Denoting

$$
\mu \equiv \frac{M_{\mathrm{BH}}}{M_{\mathrm{config}}}
$$

we see that in order to argue for the formation of a naked singularity, we must show that $\mu>1$. This indicates that the configuration does not have enough energy to collapse into a black hole which would be sufficiently large to cloak the singularity. Comparing (3.2) with (3.5), we obtain

$$
\mu>3 \phi_{0}^{2 / 3}
$$

11 An argument of this type is presented in Appendix B. 
In the region where (3.4) is applicable the lower bound in (3.7) gives $\mu \ll 1$ and the black holes are energetically allowed to form.

The lower bound (3.4) is clearly crucial in establishing possible situations where naked singularities can form. We have explored several ways to improve (3.4). The best result we have found makes use of Raychaudhuri's equation for geodesic congruences.

Consider an outgoing congruence of null geodesics emanating from the initial data surface. If the size of this congruence (the proper size of the spheres at the given $r$ and $t$ ) starts to decrease along the null geodesics, then Raychaudhuri's equation shows 12 that the geodesics must terminate at a finite affine parameter, ending in a singularity 13 . Now study outgoing null geodesics starting from larger and larger $r$ at $t=0$. If the proper size reaches a maximum at a finite affine parameter (indicating the formation of a trapped surface), then these geodesics must terminate at a singularity. Geodesics that do not enter the trapped region are likely to continue to expand and reach the boundary. Hence for some critical starting radius there will be a geodesic congruence which skirts the boundary of the trapped region within the homogeneous domain of dependence where it can be easily calculated. The largest proper size of this congruence within the homogeneous domain of dependence will give a lower bound for the size of the singularity. The details of this estimate are discussed in Appendix B.5. Numerical solution of the homogeneous FRW equations for $\phi_{0} \ll 1$ gives the result

$$
R_{s}>0.58 R_{0} \phi_{0}^{1 / 2}, \quad \text { for } \quad \phi_{0} \ll 1
$$

For small $\phi_{0},(3.8)$ is parametrically larger than (3.4), and hence this will make $\mu$ much larger than before. Specifically, computing $\mu$ with this estimate yields

$$
\mu>0.34
$$

This is independent of $\phi_{0}$ for $\phi_{0} \ll 1$ and is rather close to unity! The black hole barely has enough energy to form. If the true size of the singular region $R_{s}$ were required by the dynamics to be merely $\sim 30 \%$ larger than the bound (3.8), then naked singularities would

12 This relies on the fact that the scalar field stress tensor satisfies the null energy condition, despite the potential (2.3) being negative definite.

13 In general, the geodesics could merely caustic; but for the spherical congruence we are considering this would require the sphere to shrink to zero size, which in the present context would still imply a singularity. 
be required to form. Numerical simulations of [25] suggest that, at least in the regimes considered, this does not happen: the size of the singularity roughly matches our lower bound estimates.

At large $\phi_{0}$, the estimate for $R_{s}$ saturates at ( $c f$. Appendix B.5)

$$
R_{s}>\sigma_{s} R_{0}=0.577 R_{0}, \quad \text { for } \quad \phi_{0} \gg 1
$$

For the initial configuration (2.4), the large $\phi_{0}$ energy is (see Appendix A.1, Eq.(A.16))

$$
M_{c}=10.2 \phi_{0}^{2} R_{0}^{4}, \quad \text { for } \quad \phi_{0} \gg 1
$$

Computing the mass ratio, we find

$$
\mu>\frac{0.32}{\phi_{0}^{2}}
$$

which is much smaller than one.

The configuration (2.4) is close to the lowest energy configuration subject to boundary conditions for $\phi_{0} \ll 1$. However, this is no longer the case for large $\phi_{0}$ (despite $\frac{1}{r^{2}}$ being the slowest normalizable asymptotic fall-off). Since we would like to find a regime wherein we could argue that the initial configuration is too light to form a black hole cloaking the singularity, it is clearly desirable to start with the lowest possible energy configuration for given $R_{0}$ and $\phi_{0}$. Hence we now consider the situation when $\phi_{0} \gg 1$ more carefully. In this case we see from (3.10) that the size of the black hole that needs to form is independent of $\phi_{0}$. In choosing (2.4) we are paying an enormous price in terms of energy for large $\phi_{0}$, see (3.11). However, we can construct configurations whose energies are independent of $\phi_{0}$ very easily. Instead of having the scalar field drop to zero very slowly as in (2.4), we pick a rapid fall-off, say

$$
\phi(r)=\phi_{0} \equiv \frac{A}{R_{0}^{n}} \quad r \leq R_{0}, \quad \phi(r)=\frac{A}{r^{n}} \quad r>R_{0} .
$$

The calculation of the mass for this profile is outlined in Appendix A. We find that taking $n \rightarrow \infty$, with $\phi_{0}$ fixed, yields

$$
M_{\text {config }}=3 \pi^{2} R_{0}^{2}\left(1+R_{0}^{2}\right)
$$

which is the mass of a AdS-Schwarzschild black hole with size $R_{0}$ (this statement is true 
for all values of $\left.\phi_{0}\right)$ 14. In the large field limit making use of (3.10), we see that

$$
\mu=\frac{R_{s}^{2}\left(1+R_{s}^{2}\right)}{R_{0}^{2}\left(1+R_{0}^{2}\right)} \sim(0.58)^{4}=0.111
$$

This step function configuration may not be the minimum energy configuration for large $\phi_{0}$. However, it illustrates the important fact that even for strong fields black holes are not parametrically lighter, unlike the case with (2.4). It might indeed be possible that a case can be made for naked singularity formation by using the true minimum energy configuration along with a tighter lower bound than (3.8) and (3.10) for the size of the requisite black hole.

Hence, we see that initial $\phi$ profiles containing a flat region to allow study of singularity formation, favor formation of black holes big enough to cloak singular regions (of the lower bound size we established) in all regimes we could analyze. However, this does not necessarily imply that the exact dynamical evolution of the initial data will not create a naked singularity. One way this can be studied is by a careful numerical evolution of the data. Significant progress in such numerical evolution has been made in [15], though the regime of $R_{s}$ they used is not the most optimal for forming naked singularities.

Given that the 5-dimensional setup discussed above did not yield kinematic proof for formation of naked singularity, one might hope that we could do better in a different number of dimensions. Unfortunately this does not seem to be the case (see Appendix B).

\section{AdS/CFT with a cut-off}

In the previous section we saw that the problem with using the bulk ADM energy for (2.4) (which was negative) was its non-conservation. The conserved energy did not prevent the formation of black holes that cloak the singularity. A different possibility raised by HHM [14](v2), is to consider a cut-off AdS geometry where one imposes boundary conditions for the fields at the cut-off surface $r=R_{1}$ for all time. Then the bulk ADM energy is conserved, and one can use it to study the possibility of forming naked singularities.

14 The strict $n \rightarrow \infty$ limit results in the field profile being a step function. This case can be visualized as a thin domain wall junction between an AdS-Schwarzschild black hole of size $R_{0}$ and a FRW cosmology with scale factor set by $\phi_{0}$; a generalization of the "Wheeler bag of gold" to asymptotically AdS spacetimes. 


\subsection{Weak fields in the cut-off geometry}

As in [14], we consider the scalar-gravity system (2.2), in $\mathrm{AdS}_{5}$ with 150 $\phi\left(R_{1}\right)=\phi_{1}$ for all time. We will attempt to find solutions where $\phi(r) \ll 1$ so a linearized analysis will be valid. Since $\phi(r)$ has Dirichlet boundary conditions at finite $r$, both the normalizable mode of the linearized field equation, $\phi(r) \sim 1 / r^{2}$, and the non-normalizable mode, $\phi(r) \sim \log (r) / r^{2}$ contribute. The non-normalizable mode approaches the boundary more slowly and, roughly speaking, has less energy than the normalizable mode. To begin we choose $\phi_{0}$ and $\phi_{1}$ to pick out just the non-normalizable mode $\frac{16}{6 .}$., [14]:

$$
\begin{aligned}
& \phi(r)=\phi_{0}, \quad r \leq R_{0} \\
& \phi(r)=\frac{\phi_{1} R_{1}^{2}}{\ln R_{1}} \frac{\ln r}{r^{2}}, \quad R_{0}<r \leq R_{1} .
\end{aligned}
$$

The ADM mass $M_{\text {config }}$ of the configuration (4.1) can be computed using the constraint equation (A.4). For $\phi_{0} \ll 1$ we find

$$
M_{\text {config }}=2 \pi^{2} \phi_{0}^{2} R_{0}^{4}\left(-\left(\frac{\ln R_{1}}{\ln R_{0}}\right)^{2}+\frac{\ln R_{1}}{2\left(\ln R_{0}\right)^{2}}+\frac{1}{2}\right)
$$

We should compare this with the energy required to form a black hole of size $R_{s}$. Due to the new boundary conditions, the black hole in question is not the usual SchwarzschildAdS black hole, (since the Schwarzschild-AdS black hole would not admit static scalar field outside the horizon), but rather an AdS scalar hair black hole [26]. For weak fields outside the black hole we can use a linearized approximation and ignore backreaction. The boundary conditions on the scalar field are imposed at $R_{1}, \phi\left(R_{1}\right)=\phi_{1}$ and at the horizon $r=R_{s}$. From the field equations (A.22) and the fact that $f\left(R_{s}\right)=0$ we derive the linearized horizon boundary condition $\phi\left(R_{s}\right)+R_{s} \phi^{\prime}\left(R_{s}\right)=0$. A linear combination of the two modes that satisfies the boundary conditions is

$$
\phi_{b h}(r)=\alpha_{b h} \frac{1}{r^{2}} \ln \left(\frac{r e}{R_{s}}\right),
$$

where $\alpha_{b h}$ is determined by the boundary condition at $R_{1}$. We find (for convenience taking $\left.R_{1} \gg R_{0} \gg R_{s} \gg 1\right)$

$$
\alpha_{b h}=\frac{\phi_{0} R_{0}^{2}}{\ln R_{0}}\left(1-\frac{\ln \left(R_{s} / e\right)}{\ln R_{1}}\right)^{-1}
$$

15 To ensure that we have a sufficiently large region of AdS geometry, we choose $R_{1} \gg R_{0} \gg 1$.

16 Note that this or any configuration does not smoothly allow the cut off to be taken away, unlike the situation discussed in the previous section. 
Note that the coefficient (4.4) of the $\ln (r) / r^{2}$ fall off is slightly larger than in (4.1). This means that the black hole with the same $\phi_{1}$ has a little more scalar hair than the initial configuration. The black hole field profile is not required to go through $\phi_{0}$ at $R_{0}$ and so can become larger to minimize its energy. We see this by computing the black hole energy. As explained in Appendix A.3, we find

$$
\begin{aligned}
M_{S B H}=3 & \pi^{2} R_{s}^{2}\left(1+R_{s}^{2}\right) \\
& +2 \pi^{2} \phi_{0}^{2} R_{0}^{4}\left(1-\frac{\ln \left(R_{s} / e\right)}{\ln R_{1}}\right)^{-2}\left[-\left(\frac{\ln \left(e R_{1} / R_{s}\right)}{\ln R_{0}}\right)^{2}+\frac{\ln R_{1}}{2\left(\ln R_{0}\right)^{2}}\right] .
\end{aligned}
$$

This reduces in the large $R_{1}$ limit to

$$
M_{S B H}=3 \pi^{2} R_{s}^{2}\left(1+R_{s}^{2}\right)+2 \pi^{2} \phi_{0}^{2} R_{0}^{4}\left(-\left(\frac{\ln R_{1}}{\ln R_{0}}\right)^{2}+\frac{\ln R_{1}}{2\left(\ln R_{0}\right)^{2}}\right) .
$$

Using the previously derived estimate (3.8) for the minimum Schwarzschild radius necessary to cloak the singularity we find

$$
M_{S B H}-M_{\text {config }}=-0.66 \pi^{2} \phi_{0}^{2} R_{0}^{4}
$$

So we see that black holes which can cloak the singularity cannot be excluded from forming 17 . To check the validity of the linearized approximation we compute $\phi\left(R_{s}\right)=$ $1 / \log R_{0} \ll 1$. So we can have a large singular region and a controlled linearized analysis at the same time. This would not be the case with the weaker lower bound (3.4) .

We have studied other initial $\phi$ profiles within the linearized regime and have not found a case where naked singularities were required to form. The well behaved nature of weak field initial conditions makes us suspect that they are not the best candidates for finding a violation of these lower bound estimates. So we now turn to the nonlinear regime where $\phi$ need not be small.

\subsection{Minimal energy configurations at strong field}

To find a minimum energy initial configuration in general we need to minimize the mass function $m(r)$ with respect to the profile $\phi(r)$. While it is not difficult to derive the Euler-Lagrange equations (by taking a variation of (A.5)), it helps to think of the

17 Our results differ from those in 14 (v2) because we employ the optimal scalar hair configuration. 
coupled gravity-scalar system minimize the Einstein-Hilbert action (see Appendix A.2). To specify the problem completely we need boundary conditions; we will require that we have a homogeneous central region of size $R_{0}$ and $\phi\left(R_{0}\right)=\phi_{0}$. This determines $m\left(R_{0}\right)$ for us; the mass is just the contribution from the effective cosmological constant. Our final boundary condition is the Dirichlet boundary condition at the cut-off surface $\phi\left(R_{1}\right)=\phi_{1}$. So specification of $\left(R_{0}, \phi_{0}, R_{1}, \phi_{1}\right)$ along with the Einstein's equations for static, spherically symmetric profiles is a complete specification of the minimization problem. It should be no surprise that the equations of motion for the initial configuration are identical to that of the scalar hair black hole (which of course has different boundary conditions).

We find the dynamics simplifies in the strong field regime, $\phi_{0} \gg 1$. In this limit the coupled equations have a universal attractor solution $\phi_{a t t}(r)=\sqrt{3} \log \left(R_{1} / r\right)+\phi_{1}$ that obeys the boundary condition at $R_{1}$. This means that the minimum energy initial configuration and the scalar hair configuration have the same functional form for $r>R_{0}$. In particular, the scalar hair outside the black hole horizon $R_{s}$ has the form $\phi_{s b h}(r)=\phi_{a t t}(r)$, $r>R_{s}$. The initial profile is given by

$$
\phi(r)=\phi_{0}=\phi_{s b h}\left(R_{0}\right), \quad r \leq R_{0}, \quad \& \quad \phi(r)=\phi_{s b h}(r) \quad R_{0}<r \leq R_{1}
$$

We find in Appendix A.2 that the mass difference between this optimal initial configuration and the scalar hair black hole of size given by the bound $R_{s}>\sigma_{s} R_{0}$ with $\sigma_{s}=.577$ (equation (3.10)), is given by 18

$$
M_{S B H}-M_{\text {config }}=\frac{\pi^{2}}{3} \sigma_{s}^{2} R_{0}^{4}\left(4 \sigma_{s}^{3}-1\right) e^{\frac{2}{\sqrt{3}} \phi_{s}} e^{-\frac{1}{3} \int_{R_{0}}^{R_{1}} x \phi_{s b h}^{\prime}(x)^{2}}<0 .
$$

Again naked singularities are not required to form. One can see that the critical value of $\sigma_{s}$ for guaranteeing naked singularity formation is $\sigma_{s}^{*}=\frac{1}{4^{1 / 3}} \approx 0.63$. This implies that only a $10 \%$ increase in our estimate for the size of the black hole would be sufficient to rule out its formation on kinematic grounds.

\subsection{Field theory perspective}

So far we have focused on the 5D cut-off supergravity system to ask whether naked singularities appear to form generically. But in the full 10D theory new issues emerge. This cut-off theory turns out to be unstable 19 (see for example, 27]). First, note that (4.1) (and

18 This estimate assumes, for simplicity, that $R_{0}, R_{1}$ are fixed and $\phi_{0} \rightarrow \infty$.

19 We thank Ofer Aharony and Matt Strassler for enlightening us on this point. 
others like (4.8)) correspond to deforming the boundary theory by a relevant operator of mass dimension $\Delta=2 ; \mathcal{O}_{2}=\operatorname{Tr}\left(X^{i} X^{j}\right)-\frac{1}{6} \delta^{i j} \operatorname{Tr}\left(X^{2}\right)$, where $X^{i}$ are the adjoint scalars in the $\mathcal{N}=4$ Super Yang-Mills multiplet, transforming in the vector representation of the R-symmetry group $S O(6)$. The operator $\mathcal{O}_{2}$ is in the traceless symmetric two-tensor representation of $S O(6)$. Because of the symmetrization and trace removal, this operator has negative eigenvalues. These negative eigenvalues induce tachyonic mass terms for the adjoint scalars. Hence $\mathcal{N}=4 \mathrm{SYM}$ on $\mathbf{R}^{4}$ deformed by $\mathcal{O}_{2}$ is unstable.

We are interested in the field theory on $\mathbf{S}^{3} \times \mathbf{R}$, since we wish to work with global coordinates. There is a crucial distinction here arising from the curvature $\mathcal{R}$ of the boundary. The $\mathcal{N}=4$ Hamiltonian on $\mathbf{S}^{3} \times \mathbf{R}$ has positive mass terms for the adjoint scalars from the conformal couplings to the background curvature. In particular, the Hamiltonian for the deformed theory is schematically

$$
\mathcal{H}=\mathcal{H}\left(\mathbf{R}^{4}\right)+\mathcal{R} \operatorname{Tr}\left(X^{2}\right)+\hat{\alpha} \mathcal{O}_{2}
$$

We see that the theory defined by $\mathcal{H}$ is stable as long as $\alpha=\hat{\alpha} / \mathcal{R}<1$. We can read off $\alpha$ from the bulk initial $\phi$ configuration at large $r$. It is just the coefficient of the $\log r / r^{2}$ asymptotic fall-off. For optimal weak field scalar profile (4.1), we find $\alpha=\phi_{0} R_{0}^{2} / \ln R_{0}$. For any fixed $\phi_{0} \ll 1$ as the size of the flat region $R_{0}$ grows $\alpha$ becomes large and the theory becomes unstable. There are very weak field configurations with finite singular regions that do remain stable, though. Using the lower bound (3.8) we can write $\alpha \sim R_{s}^{2} / \ln R_{0}$ which can be made small for fixed large $R_{s}$. The generic strong field configuration is also unstable, although the profile can be fine tuned to make $\alpha$ vanish. So overall, it seems likely to us that the best possibilities for finding naked singularities will have unstable CFT duals.

\subsection{Supergravity manifestation of CFT instability}

Our argument for the instability of the gauge theory defined in (4.10), was based on our expectation from perturbative gauge theory. Understanding what this means for the dual supergravity theory is quite instructive 20 .

20 We have already seen that $\mathcal{N}=4 \mathrm{SYM}$ on manifolds with non-trivial curvature has adjoint scalars conformally coupled to the background curvature. Formally, the Hamiltonian (4.10) is analogous to the case where the manifold has negative curvature. In the latter case that analysis of [28] tells us that there is a non-zero probability for nucleating BPS D3-branes which are pushed off to the boundary of AdS. 
We expect that the theory deformed by (4.10) has a dual supergravity geometry, where BPS D3-branes have a potential that drives them to the boundary of the AdS geometry. For $\mathcal{N}=4 \mathrm{SYM}$ deformed by $\mathcal{O}_{2}$ on $\mathbf{R}^{4}$, this was studied in [27], where bulk equations were solved to find the supergravity dual. Furthermore, the probe D3-brane potential was evaluated and shown to be unbounded from below. We now extend this analysis to global coordinates.

To compute the probe potential, we need to know the full ten-dimensional geometry that is dual to the deformed field theory. Thus far we have been dealing with fivedimensional gauged supergravity with a single scalar field. Happily for us, there exists a unique lift of five dimensional solutions to ten-dimensions, via the magic of consistent truncation [21]. In practice we need to consider a static, spherically symmetric ansatz for the five-dimensional metric and solve the coupled scalar-gravity system of (2.2). However, to get insight into the probe potential, it suffices to work in a linearized approximation about the AdS background. This lets us obtain the potential felt by a D3-brane, whose world-volume is $\mathbf{S}^{3} \times \mathbf{R}$, as a function of the radial coordinate $r$ and the directions on the $\mathbf{S}^{5}$. Parameterizing the $\mathbf{S}^{5}$ as in (C.2) we find (see Appendix C for details):

$$
V_{\text {probe }}=\frac{1}{2} r^{2}\left(1-\frac{\alpha}{2 \sqrt{3}}\left(3 \sin ^{2} \xi-1\right)\right), \quad \text { for } r \gg 1 .
$$

In deriving (4.11), we assume that the supersymmetric D3-brane saturates the BPS bound.

It is not surprising that this potential is identical to the tree-level potential in the SYM action because large radius corresponds to working near the UV fixed point of the boundary field theory. As advertised, there is a critical value $\alpha_{c}=2 \sqrt{3}$ above which the probe potential is unbounded below. We have computed the asymptotic form of the potential; it is possible that the true potential has a local minimum in the interior of the geometry. This however will not help stabilize the theory in the high energy regime, as the branes can be excited out of the well to infinity. The behavior of probe branes in the dual supergravity justifies our fears about the CFT being ill-defined in the regime $\alpha>\alpha_{c}$.

Apart from this instability, there is general concern with the above analysis. In the context of AdS/CFT radial evolution corresponds to RG evolution in the boundary theory. In particular, moving toward the center of AdS (i.e., small $r$ ) corresponds to the IR of the dual field theory. Perturbations of any field theory by a relevant operator grow in the IR, possibly causing drastic modifications, which are reflected in the supergravity dual. In many examples one manifestation of these effects is a static naked singularity at finite $r$ 
in the 5D truncated gravity theory $(c f .,[27)$. But in 10D the effects often take a different form, often involving brane dynamics (see for example [29], [30]). The breakdown of the linearized approximation in the 5D supergravity indicates that the effect of $\mathcal{O}_{2}$ are getting large. So there may well be new dynamical channels available for the behavior of the 10D theory. In order to fully understand its behavior these effects must be incorporated.

\section{A stable example in 3 dimensions}

It is important to know if there are any examples where the underlying theory is stable and naked singularities are produced. It appears there may be such an example in $d=3$.

For scalar fields that saturate the BF bound, the story is very similar in all dimensions $d \geq 4$. In supergravity backgrounds of the form $\operatorname{AdS}_{d} \times \mathbf{S}^{q}$, there are scalar fields saturating the $\mathrm{BF}$ bound $m_{B F}^{2}=-\frac{(d-1)^{2}}{4}$, for odd $q>3$ [31]. This scalar field is a particular linear combination of the graviton mode and the RR-field, polarized in the $\mathbf{S}^{q}$, with its wavefunction being a spherical harmonic of angular momentum $l=(q-1) / 2$ on $\mathbf{S}^{q}$. Since the spherical harmonics are non-positive definite, the operator dual to the field has negative eigenvalues. Similar statements can be made if we replace the $\mathbf{S}^{q}$ by some other compact Einstein manifold. In all these situations the dual CFT will suffer from an instability.

The situation in $\mathrm{AdS}_{3}$ is however very different. For concreteness we will concentrate on the case of $\mathrm{AdS}_{3} \times \mathbf{S}^{3} \times X$, where $X$ may be $T^{4}$ or $K 3$. In this case the BF scalars do not arise from modes of the graviton on the $\mathbf{S}^{3}$. They come from KK-reduction of anti-self dual tensor fields in six-dimensional supergravity 21 [32], [33]. These scalars are expected to be dual to fermion bilinears with $\Delta=1$ in the dual CFT [32].

Since the dual operators are fermion bilinears, we might hope that the deformed boundary theory is stable. But, it is plausible that the nature of the anti self-dual tensor field's wavefunction on the $\mathbf{S}^{3}$ (it is a $l=1$ spherical harmonic) may result in an instability, which in this case would be manifested by nucleating BPS D-strings. Nonetheless, there is a way to stabilize the theory on the boundary. We can consider the boundary CFT at a point in moduli space ( $c f$. [34], [28] for discussions of the moduli space) where the marginally bound BPS objects are lifted from the spectrum.

21 Type IIB theory on $X$ is described by a six dimensional low energy effective lagrangian, whose field content is the super-graviton multiplet and $n$ tensor multiplets, with $n=5$ for $T^{4}$ compactification and $n=21$ for $K 3$. The anti self-dual tensor fields are part of the tensor multiplet. 
Recall that the geometry $\mathrm{AdS}_{3} \times \mathbf{S}^{3} \times X$ is the near-horizon geometry of the D1-D5 system, with the D5-branes wrapping $X$. If we turn on NS-NS fluxes through $X$, then we will resolve the small instanton singularity and make the D1-D5 system truly bound, as opposed to marginally bound as in the absence of the fluxes. Then a probe D-string feels a force [35], which results in a positive definite potential. The deformation caused by the operator adds a subleading contribution which could be negative, but the net effect would be such as to stabilize the system.

Schematically, the potential will now look like

$$
V_{\text {probe }}=B r^{2}-\alpha \log (r), \quad \text { for } r \gg 1
$$

The leading term $B r^{2}$ is a result of turning on fluxes through the internal space. In case of the probe D3-brane calculation outlined in Appendix C, this term maps to the $r^{4}$ contribution that canceled between the DBI and the WZ contributions to the potential. The subleading term is proportional to $\alpha$ (upto order 1 coefficients) and is a direct consequence of the deformation of the CFT. Note that in this case the boundary CFT lives on $\mathbf{R} \times \mathbf{S}^{1}$, the boundary of $\mathrm{AdS}_{3}$ and hence we do not get a mitigating contribution from the curvature coupling. We note in passing that a very similar ideas were explored in [36], in the context of multi-boundary Euclidean-AdS geometries.

We are now in a position to propose a scenario which does not suffer from instabilities. The set-up we would like to propose has 3-dimensional gravity coupled to a single scalar field $\phi$, with some scalar potential $V_{3}(\phi)$.

$$
S_{3}=\int d^{3} x \sqrt{g}\left(\frac{1}{2} R-\frac{1}{2}(\nabla \phi)^{2}-V_{3}(\phi)\right)
$$

The exact scalar potential $V_{3}(\phi)$ can in principle be extracted from [37]. For our purposes a quadratic approximation will suffice: $V_{3}(\phi)=-1-\frac{1}{2} \phi^{2}$. Ideally we should check that the scalar $\phi$ does not source other three dimensional fields to ensure consistency of the truncated lagrangian (5.2). We will assume that this is true.

For a scalar of mass $m^{2}=m_{B F}^{2}=-1$, the Klein-Gordon equation on $\mathrm{AdS}_{3}$ background tells us that $\phi(r) \sim C_{1} / r+C_{2} \log (r) / r$ at large $r$. The arguments presented in Section 3 will continue to apply in this case as mentioned before. Configurations analogous to (4.1) and (4.8) will as in five dimensions continue to have enough energy for the formation of black holes. As in those cases it may be possible to rule out black hole formation by getting a more accurate lower bound on its size. 
This configuration is expected to be dual to a well behaved two-dimensional QFT. From the form of the probe potential (5.1), it is possible that there is a global minimum at some finite $r$. This can be avoided by tuning the fluxes so that the D-strings are stabilized at some small radial distance, so that there is definitely a regime in the domain

of dependence of the region $r \leq R_{0}$ on the initial data slice, where the formation of a naked singularity is a possibility.

The value of the scalar field is large at $R_{0}$ here as well, so the effect of the relevant operator is large. So the boundary CFT vacuum structure and the 10D bulk theory is dramatically altered at small $R$ (in the IR). These dramatic alterations may alter the prospects for naked singularity formation in the full 10D theory. One concrete effect we can point to is that the relevant operator breaks supersymmetry, and so will induce a potential for the various moduli. This might make the values chosen to stabilize the branes unstable.

\section{Discussion}

The history of cosmic censorship conjecture has been characterized by many plausible counter-examples, most of which on close examination have proved to be incorrect. The fascinating aspect of the HHM proposal is that it is based on an extremely simple kinematic argument, which essentially says that in the configurations of interest there is not enough energy to cloak the putative singularity by a black hole horizon.

The proposal of HHM for cosmic censorship violation in asymptotically AdS spacetimes with general scalar potential is attractive, but there remains a gap in the arguments as we illustrate numerically in Appendix B.6 and has been independently discussed in [16]. The HHM proposal in the AdS/CFT context is particularly interesting, though, because of the potential for learning about stringy singularity resolution.

Within 5D supergravity, HHM discussed two distinct scenarios to probe the formation of naked singularities. The first one, in infinite volume AdS, suffers from the problem of the bulk energy not being conserved, and the conserved energy being sufficient for the formation of a black hole cloaking the singularity. This does not necessarily mean that a black hole will form. It is possible that the lower bound on the size of the singular region used in these estimates is significantly exceeded in the exact dynamics. Or the singularity could occur before the black hole has a chance to form. Numerical evolution of the field equations is one promising way to address these possibilities. The numerical study carried out recently in [15] indicates that evolution of the HHM data results in the formation of 
a trapped surface, pointing toward black hole horizon formation. This is suggestive, but because trapped surfaces are ubiquitous in the central region it will be important to follow the evolution further and see an external black hole geometry form. Numerical studies are also being carried out by [25] (using coordinates that are regular at the horizon) which suggest black hole formation; in fact, the final size of the singularity is approximately equal to our analytic lower bound estimate.

In the alternate scenario of cut-off AdS/CFT we have seen again that black hole formation is kinematically possible and we cannot argue that naked singularities must form. It is possible that they do form, though, for reasons mentioned above.

However, for the most likely initial data for singularity formation, the full 10D theory with cut-off suffers from an instability: D3 brane shells expand outward to the AdS boundary. This is clear from the boundary gauge theory point of view and from a bulk D-brane probe calculation.

Let us assume for a moment that a naked singularity does form in the AdS/CFT context. Assuming the 5D supergravity is a consistent truncation of 10D supergravity, we can lift the 5D naked singularity solution to an exact naked singularity solution of $10 \mathrm{D}$ supergravity. The central question would now become, what resolves this singularity? One possibility is that stringy effects do so. This is presumably the case for the nongeneric Choptuik [4] type naked singularities. These are, roughly speaking, the threshold formation of an arbitrarily small Schwarzschild black hole. This should be resolved in string theory by the correspondence between very small black holes and highly excited strings [38], [39]. It is clear that any such resolution of the singularities discussed by HHM will involve the dynamics of D3-branes in 10D. The work of [29], 27] make clear that the static naked singularities in these 5D supergravities are dimensionally reduced images of D3-brane distributions in 10D. More generally we have come to expect naked singularities in lower dimensional theories to have explanations in terms of brane dynamics in the full $10 \mathrm{D}$ theory [40], 30].

There are other issues to consider in the cut-off HHM context. The instability of this theory is also due to brane dynamics. It should be visible in the classical 10D supergravity equations because black 3-branes with finite horizon size can be described there 22 . It is

22 There is a subtlety here. The black 3-brane of finite horizon size is not BPS and so the stabilizing $r^{4}$ term in the probe brane potential is present. But in the strong field regime and horizon sizes of AdS scale the stable brane position will be at $r \gg R_{0}$ 
possible that with generic 10D initial data such branes will be created and then move to the boundary 23. This will decrease the energy and RR charge, resulting in a space of higher and higher negative curvature, presumably ending in a completely singular geometry. From the boundary CFT point of view, as D-branes move to the boundary, the effective $N$ of the $S U(N)$ theory decreases, resulting in a smaller AdS radius. The endpoint is $N \sim 1$. In any event, the true long time behavior of the system will be dominated by this instability.

The other possibility is that stringy and/or quantum effects near the singularity are required to create D-branes that then move to the boundary. The production would presumably continue until all D-branes move to the boundary and the whole space becomes singular.

Finally, we saw that there may well be a stable $\mathrm{AdS}_{3} / \mathrm{CFT}_{2}$ example. In this case we have argued that the rich moduli space of the dual CFT may be put to use in stabilizing the theory. Here we will have to numerically investigate the scenario to check whether we have a good kinematic argument for naked singularity formation. But here (as in the 5D case) the relevant perturbation will have a large IR effect which could dramatically affect the discussion of the fate of the singularity.

An interesting question which we have not addressed in this paper concerns the signature of naked singularities that might be seen in the dual gauge theory. A priori it seems clear that the signatures ought to be more marked and easier to access than those of the black hole singularities as studied in [13]. The formative stages of a naked singularity will be characterized by increase in curvatures and local correlators on the boundary should be sensitive to these effects. One might speculate that the formation of the singularity would be characterized by some spiky behaviour in the boundary correlators which would be smoothed out upon inclusion of quantum effects.

A related question concerns the signals of an "almost" naked singularity. We have found a number of examples where if the singularity is cloaked at all, the maximum proper time between the horizon and the singularity will be much less than one AdS time. This almost naked singularity should have an impressive signature to outside the horizon observers - presumably large curvature oscillations that eventually settle down to the black hole geometry. These oscillations should contain large amounts of information about the singularity.

23 The behavior near the singularity will be modified, but not made nonsingular, by the lift to 10D supergravity. A transition to chaotic BKL type behavior is expected [41]. 
A probe of the kind discussed in [13] may well be useful here, at least for analytic initial data. The signal of the behind the horizon singularity discussed there was a pole on the second sheet of a correlation function, a bit like a broad resonance. Perhaps as the black hole singularity comes closer and closer to the horizon the resonance becomes narrower and narrower, possibly even emerging from behind the horizon onto the first sheet.

\section{Acknowledgments}

We would like to thank Ofer Aharony, Andrei Frolov, Michael Gutperle, Thomas Hertog, Gary Horowitz, Petr Hořava, Shamit Kachru, Per Kraus, Juan Maldacena, Simon Ross and Matt Strassler for useful discussions. This work is supported in part by NSF grants PHY-9870115 and PHY-0098840, DOE grant DE-AC03-76SF00098, the Stanford Institute for Theoretical Physics and the Berkeley Center for Theoretical Physics.

\section{Appendix A. Masses of various configurations}

\section{A.1. Power law fall-off profiles in five dimensions}

Consider the lagrangian (2.2). We are interested in picking some initial data slice, a spacelike hypersurface $\Sigma$ at $t=0$, and evolve the data prescribed on this surface forward in time. We choose time symmetric configurations which allow us to set all time derivatives to zero. Einstein's equations projected onto $\Sigma$ give us the constraint equations that the data is required to satisfy. For (2.2) the equations read:

$$
{ }^{(4)} \mathcal{R}=g^{i j} \partial_{i} \phi \partial_{j} \phi+2 V(\phi)
$$

where $g_{i j}$ is a Euclidean metric on the surface $\Sigma$ and ${ }^{(4)} \mathcal{R}$ the associated Ricci scalar. For spherically symmetric configurations we can parametrize the metric on $\Sigma$ as

$$
d s_{\Sigma}^{2}=\left(1-\frac{m(r)}{3 \pi^{2} r^{2}}+r^{2}\right)^{-1} d r^{2}+r^{2} d \Omega_{3}^{2}
$$

For this geometry the ADM mass is defined to be

$$
M_{\text {config }}=\lim _{r \rightarrow \infty} m(r) .
$$


From the constraint equation (A.1), we find:

$$
m_{, r}+\frac{1}{3} r\left(\phi_{, r}\right)^{2} m=2 \pi^{2} r^{3}\left[V(\phi)+6+\frac{1}{2}\left(1+r^{2}\right)\left(\phi_{, r}\right)^{2}\right] .
$$

This can be integrated to give

$$
\begin{aligned}
& m(r)=2 \pi^{2} \frac{1}{F(r)} \int_{0}^{r} F(x) G(x) d x \\
& F(x)=\exp \left(\int_{0}^{x} d y \frac{1}{3} y(\phi, y)^{2}\right) \\
& G(x)=x^{3}\left[V(\phi)+6+\frac{1}{2}\left(1+x^{2}\right)\left(\phi_{, x}\right)^{2}\right]
\end{aligned}
$$

Before proceeding, let us write the potential (2.3) in a more useful form;

$$
\begin{aligned}
V(\phi) & =-2\left(e^{\frac{2}{\sqrt{3}} \phi}+2 e^{-\frac{1}{\sqrt{3}} \phi}\right)=\sum_{m=0}^{\infty} a_{m} \phi^{m} \\
\text { where } \quad a_{m} & =-\frac{2}{m !} \frac{1}{3^{\frac{m}{2}}}\left(2^{m}+2(-1)^{m}\right)
\end{aligned}
$$

Consider a general field profile,

$$
\phi(r)=\phi_{0} \equiv \frac{A}{R_{0}^{n}} \quad r \leq R_{0}, \quad \phi(r)=\frac{A}{r^{n}} \quad r>R_{0} .
$$

We then have

$$
F(x)=1 \quad x \leq R_{0}, \quad F(x)=\exp \left(-\frac{\zeta}{x^{2 n}}+\frac{\zeta}{R_{0}^{2 n}}\right) \quad x>R_{0},
$$

with

$$
\zeta \equiv \frac{n}{6} A^{2}
$$

and

$$
\begin{aligned}
G(x) & =x^{3}\left(V\left(\phi_{0}\right)+6\right) \quad x \leq R_{0}, \\
& =x^{3}\left(\sum_{m=2}^{\infty} a_{m} \frac{A^{m}}{x^{m n}}+\frac{n^{2} A^{2}}{2}\left(1+x^{2}\right) \frac{1}{x^{2 n+2}}\right) \quad x>R_{0} .
\end{aligned}
$$

The contribution to $G(x)$ from the $m=2$ term in the summation and the last term in (A.10) have scale together as $x^{3-2 n}$, with a coefficient $A^{2}\left(-2+\frac{n^{2}}{2}\right)$. This vanishes at $n=2$, but closer examination reveals that the integral is divergent and in fact behaves as $1 /(n-2)$. To see this, it is useful to introduce the incomplete Gamma function:

$$
\gamma(\beta, x)=\int_{0}^{x} d t e^{-t} t^{-1+\beta}
$$


This is strictly defined for $\operatorname{Re}(\beta)>0$. The $\beta \rightarrow 0$ limit is defined using confluent Hypergeometric function and one has:

$$
\lim _{\beta \rightarrow 0} \beta \gamma(\beta, x)=1
$$

Evaluation of (A.5) for (A.7) is straightforward :

$$
\begin{aligned}
\frac{1}{2 \pi^{2}} M_{\text {config }}= & -\frac{R_{0}^{4}}{2}\left[\exp \left(2 \sqrt{\frac{2}{n}} \frac{\sqrt{\zeta}}{R_{0}^{n}}\right)+2 \exp \left(-\sqrt{\frac{2}{n}} \frac{\sqrt{\zeta}}{R_{0}^{n}}\right)-3\right] \exp \left(-\frac{\zeta}{R_{0}^{2 n}}\right) \\
& +3\left[\zeta^{\frac{2}{n}}\left(\frac{1}{2}-\frac{2}{n^{2}}\right) \gamma\left(1-\frac{2}{n}, \frac{\zeta}{R_{0}^{2 n}}\right)+\frac{1}{2} \zeta^{\frac{1}{n}} \gamma\left(1-\frac{1}{n}, \frac{\zeta}{R_{0}^{2 n}}\right)\right] \\
& +\frac{1}{2 n} \zeta^{\frac{2}{n}} \sum_{m=3}^{\infty} a_{m}\left(\frac{6}{n}\right)^{m / 2} \gamma\left(\frac{m}{2}-\frac{2}{n}, \frac{\zeta}{R_{0}^{2 n}}\right)
\end{aligned}
$$

The first line is the contribution from $r \in\left[0, R_{0}\right]$. The second line is the contribution from the gradient term and the quadratic term in the potential. The last line includes the contribution from the potential. The limit $n \rightarrow 2$ is taken so as to isolate the divergence coming from the quadratic term in the potential and the $r^{2}\left(\phi_{, r}\right)^{2}$ contribution. Using (A.12) it is simple to show that,

$$
\begin{aligned}
\frac{1}{2 \pi^{2}} M_{\text {config }}=- & \frac{R_{0}^{4}}{2}\left[\exp \left(2 \frac{\sqrt{\zeta}}{R_{0}^{2}}\right)+2 \exp \left(-\frac{\sqrt{\zeta}}{R_{0}^{2}}\right)-3\right] \exp \left(-\frac{\zeta}{R_{0}^{4}}\right) \\
+3 & {\left[\zeta+\frac{1}{2} \sqrt{\zeta} \gamma\left(\frac{1}{2}, \frac{\zeta}{R_{0}^{4}}\right)+\frac{\zeta}{12} \sum_{m=3}^{\infty} a_{m}(3)^{m / 2} \gamma\left(\frac{m}{2}-1, \frac{\zeta}{R_{0}^{4}}\right)\right] . }
\end{aligned}
$$

While the above formula is ungainly it is easy to perform a small $A$ expansion and we obtain,

$$
M_{\text {config }}=\pi^{2}\left(1+\frac{2}{R_{0}^{2}}\right) A^{2}+\mathcal{O}\left(A^{3}\right)=\pi^{2} \phi_{0}^{2} R_{0}^{4}+\cdots
$$

Note that if we first set $n=2$ then the first term in the second line of (A.13) can be set to zero. This will lead to the negative energy quoted in [14], i.e., $M_{\text {config }} \approx-\pi^{2} A^{2}$.

It is also useful to consider the value of the mass for the case of large fields $\phi_{0} \gg 1$. In this case, the negative contribution from the inner region is exponentially suppressed (since $\zeta \sim \phi_{0}^{2} \gg 1$ ). Moreover, it is possible to sum the series in (A.14), and we obtain

$$
M_{\text {config }}=10.2348 \phi_{0}^{2} R_{0}^{4}+\sqrt{3} \pi^{5 / 2} \phi_{0} R_{0}^{2}+\mathcal{O}\left(e^{-\phi_{0}^{2}}\right)
$$


Another interesting limit to consider is $n \rightarrow \infty$ with $\phi_{0}$ held fixed. This limit results in the field profile being a step function. In this case from (A.13), we obtain,

$$
M_{\text {config }}=3 \pi^{2} R_{0}^{2}\left(1+R_{0}^{2}\right)
$$

which is independent of $\phi_{0}$ !

\section{A.2. Generalization to other dimensions}

In $\mathrm{AdS}_{d}$ one can parametrize a spherically symmetric Euclidean slice $\Sigma_{d-1}$ by an appropriate generalization (A.2);

$$
d s_{\Sigma}^{2}=\left(1-\frac{m(r)}{r^{d-3}}+r^{2}\right)^{-1} d r^{2}+r^{2} d \Omega_{d-2}^{2}
$$

The analog of the constraint equation (A.1) equates the Ricci scalar of $d-1$ dimensional metric (A.18) to the scalar stress tensor, resulting in

$$
m_{, r}+\frac{1}{d-2} r\left(\phi_{, r}\right)^{2} m=\frac{2}{d-2} r^{d-2}\left[V(\phi)+\frac{(d-1)(d-2)}{2}+\frac{1}{2}\left(1+r^{2}\right)\left(\phi_{, r}\right)^{2}\right] \text {. }
$$

The mass of the configuration can be obtained as before by integrating (A.19). The ADM mass for any asymptotically $\mathrm{AdS}_{d}$ configuration is given as

$$
M_{\text {config }}=\frac{1}{\Gamma\left(\frac{d-1}{2}\right)}(d-2) \pi^{\frac{d-1}{2}} \lim _{r \rightarrow \infty} m(r)
$$

\section{A.3. Scalar hair black holes}

Scalar hair black holes are solutions to the lagrangian (2.2), with a non-trivial scalar field profile outside the black hole horizon. We are interested in static spherically symmetric black holes since we imagine that the scalar configurations considered in Section 4, will ultimately settle down into a static configuration. Hence we choose the following metric ansatz:

$$
d s^{2}=-e^{-2 \delta(r)} f(r) d t^{2}+\frac{1}{f(r)} d r^{2}+r^{2} d \Omega_{3}^{2}
$$

The equations of motion following from (2.2) are:

$$
\begin{aligned}
\delta^{\prime}(r) & =-\frac{1}{3} r \phi^{\prime}(r)^{2} \\
r f^{\prime}(r)-2+2 f(r) & =-\frac{1}{3} r^{2}\left(f(r) \phi^{\prime}(r)^{2}+2 V(\phi)\right) \\
f(r)\left(\phi^{\prime}(r)+r \phi^{\prime \prime}(r)\right) & =r \frac{\partial V}{\partial \phi}+\frac{2}{3} \phi^{\prime}(r)\left(r^{2} V(\phi)-3\right)
\end{aligned}
$$


To evaluate the black hole mass, it is simpler to parametrize the function $f(r)$ in terms of the mass function $m(r)$ as

$$
f(r)=1+r^{2}-\frac{m(r)}{3 \pi^{2} r^{2}}
$$

It is simple to integrate for $\delta(r)$ and $m(r)$ in terms of the scalar profile $\phi(r)$. Since we are looking at a static spherically symmetric system, we find, not surprisingly, that $m(r)$ is given in terms of $\phi(r)$ by the now familiar equation (A.4).

In order to calculate the mass of a scalar hair black hole of horizon size $R_{s}$, we have to solve the equations (A.22) numerically. However, one can get a reasonable estimate of the mass in the weak field limit analytically. As explained in the text, we take the linearized solutions for the scalar field in AdS background and impose the appropriate boundary conditions. The correct profile in the weak field limit is as given in (4.3).

The mass of the black hole can now be computed using the formula (A.5). We have two contributions: The contribution to the mass from within the horizon can be reasonably approximated by the mass of an AdS-Schwarzschild black hole of the same size. For the region outside the horizon we take the profile to be as in (4.3). Moreover we can drop the exponential term in (A.5), i.e., set $F(x)=1$, since $\phi$ is small. We therefore have:

$$
M_{S B H}=3 \pi^{2} R_{s}^{2}\left(R_{s}^{2}+1\right)+2 \pi^{2} \int_{R_{s}}^{R_{1}} d x x^{3}\left[V(\phi)+6+\frac{1}{2}\left(1+x^{2}\right)\left(\phi_{, x}\right)^{2}\right],
$$

which integrates to

$$
\frac{M_{S B H}}{2 \pi^{2}}=\frac{3}{2} R_{s}^{2}\left(1+R_{s}^{2}\right)+\alpha_{b h}^{2}\left[-\left(\frac{\ln \left(e R_{1} / R_{s}\right)}{\ln R_{0}}\right)^{2}+\frac{\ln R_{1}}{2\left(\ln R_{0}\right)^{2}}\right] .
$$

In deriving (A.25), we work in the regime $R_{1} \gg R_{0} \gg R_{s} \gg 1$ and drop subleading terms like $1 / R_{s}$ that show up upon integration. The mass for the configuration (4.1) has been evaluated in (4.2).

\section{A.4. Strong fields in finite radius cut-off AdS}

From Appendix A.2. we learn that for the finite radius cut-off AdS space there is no parameter regime for weak fields with the scalar profile as in (4.1) where black holes formation is prohibited on simple kinematic grounds. One basic problem was that in the weak field regime the singularity forms too late, which means that the black hole that needs to form can be quite small (see Appendix B.2). We learn there that for strong fields, 
the singularity will form very quickly and in principle by going to the non-linear regime $R_{s} \sim R_{0}$. This by itself is not good enough as evidenced in (A.16) for non cut-off AdS scenario. This is because the configurations we have been considering so far are not the optimal ones. As discussed in the text it is possible to set-up a minimization problem with appropriate boundary conditions.

Since the weak field regime is not well suited to the formation of naked singularities, we would like to examine the strong field situation closely. In this case from the discussion of FRW cosmologies with strong fields we learn that the putative horizon radius is a finite fraction of the radius of the homogeneous region; $R_{s}=\sigma_{s} R_{0}$, with $\sigma_{s}=0.577$ in five dimensions (3.10) (cf., Appendix B.5).

Suppose the value of the scalar field at the horizon is $\phi\left(R_{s}\right)=\phi_{s} \gg 1$. Consider the following field redefinitions: $\psi(r)=\phi(r)-\phi_{s}$ and $m(r)=e^{\frac{2}{\sqrt{3}} \phi_{s}} \mu(r)$. Since the scalar potential is a sum of two exponentials, it takes a particularly simple form for large $\phi_{s}$; $V(\phi) \approx-2 e^{\frac{2}{\sqrt{3}} \phi_{s}} \exp \left(\frac{2}{\sqrt{3}} \psi\right)$.

The equations of motion for $m(r)$ and $\phi(r)$, A.22), become very simple in terms of the redefined fields;

$$
\begin{gathered}
\mu^{\prime}(r)+\frac{r}{3} \psi^{\prime}(r)^{2} \mu(r)=2 \pi^{2} r^{3} V(\psi)+\mathcal{O}\left(e^{-\frac{2}{\sqrt{3}} \phi_{s}}\right) \\
-\frac{1}{r^{2}} \mu(r)\left(\psi^{\prime}(r)+r \psi^{\prime \prime}(r)\right)=2 \pi^{2}\left(\sqrt{3} r+\psi^{\prime}(r) r^{2}\right) V(\psi)+\mathcal{O}\left(e^{-\frac{2}{\sqrt{3}} \phi_{s}}\right)
\end{gathered}
$$

Here $V(\psi)=-2 \exp \left(\frac{2}{\sqrt{3}} \psi\right)$ and we made use of the fact that $V_{, \psi}=\frac{2}{\sqrt{3}} V_{\psi}$. Ignoring all the terms containing the factor $\mathcal{O}\left(e^{-\frac{2}{\sqrt{3}} \phi_{s}}\right)$ for the moment, we see that there is an attractor solution to (A.27),

$$
\psi_{*}(r)=\sqrt{3} \log \left(\frac{R_{s}}{r}\right) .
$$

We picked the constant of integration to set $\psi\left(R_{s}\right)=0$ as required. This however cannot be the full story; the profile for the field $\psi(r)$ cannot decay logarithmically in $r$, for if it were, the geometry would not be asymptotically AdS. When does this profile for $\psi(r)$ break down? As can be seen from the equations it will happen when the terms multiplied by $\mathcal{O}\left(e^{-\frac{2}{\sqrt{3}} \phi_{s}}\right)$ start to become significant. This will happen at a length scale $R_{*} \sim\left(e^{\frac{1}{\sqrt{3}} \phi_{s}}\right)$. It is easy to engineer $R_{s} \ll R_{*}$ so that there is a large region where we an use (A.28). We restrict ourselves to regimes where the $r>R_{*}$ region is negligible.

With $R_{s} \ll R_{*}$ and $R_{s}=\sigma_{s} R_{0}$, it is now very easy to estimate the mass difference between the black hole and the configuration (4.8). We basically need to solve for $\mu(r)$ 
from (A.26), and in particular need the solution only in the range $r \in\left(R_{s}, R_{0}\right)$. Using (A.28), we obtain

$$
\frac{d}{d r}(r \mu(r))=-4 \pi^{2} R_{s}^{2} r^{2}
$$

For the black hole the boundary condition is $\mu_{s b h}\left(R_{s}\right)=0$. This follows because the black hole mass is independent of $\phi_{0}$ and in the scaling regime the usual factor of $3 \pi^{2} R_{s}^{2}\left(1+R_{s}^{2}\right)$ is exponentially suppressed. This implies,

$$
M_{S B H}=-\frac{4}{3} \pi^{2} \sigma_{s}^{2} R_{0}^{4}\left(1-\sigma_{s}^{3}\right) e^{\frac{2}{\sqrt{3}} \phi_{s}} e^{-\frac{1}{3} \int_{R_{0}}^{R_{1}} x \psi_{s b h}^{\prime}(x)^{2}} .
$$

On the other hand, for the configuration (4.8) we have $\mu_{\text {config }}\left(R_{0}\right)=-\pi^{2} R_{0}^{4} e^{2} \psi_{*}\left(R_{0}\right) / \sqrt{3}=$ $-\pi^{2} R_{s}^{2} R_{0}^{2}$. So we get

$$
M_{\text {config }}=-\frac{1}{3} \pi^{2} \sigma_{s}^{2} R_{0}^{4} e^{\frac{2}{\sqrt{3}} \phi_{s}} e^{-\frac{1}{3} \int_{R_{0}}^{R_{1}} x \psi_{s b h}^{\prime}(x)^{2}} .
$$

One of prefactors in the mass formulae can be traced to the scaling, while the other is related to the fact that as we go out to larger length scales the contribution to the mass from the inner regions gets exponentially suppressed as in A.5). The mass difference is now

$$
M_{S B H}-M_{\text {config }}=\frac{\pi^{2}}{3} \sigma_{s}^{2} R_{0}^{4}\left(4 \sigma_{s}^{3}-1\right) e^{\frac{2}{\sqrt{3}} \phi_{s}} e^{-\frac{1}{3} \int_{R_{0}}^{R_{1}} x \psi_{s b h}^{\prime}(x)^{2}}<0
$$

since $\sigma_{s}=0.577$.

\section{Appendix B. Field dynamics in $d$ dimensions}

We want to ask whether naked singularities are more likely to form in dimensions other than 5, and examine (for particular dimensions of interest) whether there might be numerical "windows of opportunity" for initial conditions that would lead to naked singularity. This involves 1) verifying that a homogeneous scalar field collapses to a singularity, 2) estimating the mass of a sufficiently large black hole which would cloak the singularity, and 3) estimating the mass of the starting configuration (as already discussed in Appendix A).

In Appendix B.1 we study the FRW cosmology in $d$ dimensions and show that the cosmologies big crunch in all dimensions. In B.2 we make an analytic estimate of the size of the singularity in the weak field regime. In B.3 we exhibit a scaling argument valid for 
the $5 \mathrm{~d}$ supergravity potential in the strong field regime. In B.4 we study the size of the singularity numerically, using the same physical prescription as in B.2. This allows us to get a better estimate for the numerical coefficients in the weak field limit and also understand the strong field behaviour. Finally, in B.5 we present a more sophisticated argument for determining the size of the singularity and give our numerical results. While the main focus of this paper is on cosmic censorship in the AdS/CFT context, for completeness, in B.6 we discuss the corresponding estimates in the context of the double-well potential of [7].

\section{B.1. Evolution toward singularity}

The first step is to model the evolution of a homogeneous scalar field in hyperbolic slices of AdS. This is an accurate description of the dynamics in the domain of dependence of the homogeneous region $r \leq R_{0}$ for the configurations considered in the body of the paper. The metric in $d$ dimensions can be written as

$$
d s^{2}=-d T^{2}+a(T)^{2}\left(\frac{d R^{2}}{1+R^{2}}+R^{2} d \Omega_{d-2}^{2}\right)
$$

where the scale factor $a(T)$ and the scalar field $\phi(T)$ are to be determined from the Einstein's equations $G_{\mu \nu}=T_{\mu \nu}$ (having set $8 \pi G_{N} \equiv 1$ ).

From (B.1) we can readily find the Einstein tensor:

$$
G_{T T}=\frac{1}{2}(d-1)(d-2) \frac{\dot{a}^{2}-1}{a^{2}}, \quad G_{R R}=-\frac{(d-2)}{2} \frac{2 a \ddot{a}+(d-3)\left(\dot{a}^{2}-1\right)}{1+R^{2}}
$$

(As an aside, note that the Ricci scalar is

$$
\mathcal{R}=(d-1) \frac{2 a \ddot{a}+(d-2)\left(\dot{a}^{2}-1\right)}{a^{2}}
$$

This indicates a curvature singularity as $a \rightarrow 0$, unless $a(T) \sim \cos T$ as for pure AdS, where $a=0$ corresponds merely to a coordinate singularity. But as we will see below, for nontrivial scalar field, $a$ cannot be a simple trigonometric function.) We can also find the stress tensor in terms of the (as yet unknown) potential $V(\phi)$, by evaluating $T_{\mu \nu}=\partial_{\mu} \phi \partial_{\nu} \phi-\frac{1}{2} g_{\mu \nu}\left[\partial_{\mu} \phi \partial^{\mu} \phi+2 V\right] ;$ in the present context,

$$
T_{T T}=\frac{1}{2} \dot{\phi}^{2}+V, \quad T_{R R}=\frac{a^{2}}{1+R^{2}}\left(\frac{1}{2} \dot{\phi}^{2}-V\right)
$$


Putting (B.2) and (B.4) together, we obtain

$$
\begin{gathered}
\dot{a}^{2}-\frac{2}{(d-1)(d-2)} a^{2}\left(\frac{1}{2} \dot{\phi}^{2}+V\right)=1 \\
\frac{\ddot{a}}{a}-\frac{2}{(d-1)(d-2)}\left(V-\frac{(d-2)}{2} \dot{\phi}^{2}\right)=0
\end{gathered}
$$

Finally, from the stress tensor conservation $\nabla^{\mu} T_{\mu \nu}=0$, we can derive the (linearly dependent) third equation

$$
\ddot{\phi}+(d-1) \frac{\dot{a}}{a} \dot{\phi}+V_{, \phi}=0
$$

Evolving these equations is difficult to do analytically, but it is relatively straightforward numerically - once we know the full $V(\phi)$. Finding $V(\phi)$ suited for the present purposes is however often prohibitively difficult. Nevertheless, there are 2 regimes where we can make progress: Firstly, for small fields, we can use the quadratic expansion of $V$ for a BF-saturating field; in particular, the constant term is fixed by the AdS radius (which we'll set $=1$ ) and the quadratic term by $m_{B F}^{2}=-\frac{(d-1)^{2}}{4}$. Thus, $V(\phi)=-\frac{(d-1)(d-2)}{2}-\frac{(d-1)^{2}}{8} \phi^{2}+\mathcal{O}\left(\phi^{3}\right)$. Numerically evolving $\phi(T)$ and $a(T)$ in this potential leads to a curvature singularity at $T<\frac{\pi}{2}$ (though of course in the process $\phi \rightarrow \infty$ and the approximation to $V$ is no longer valid).

Secondly, however, we can study the near-singularity regime, where the potential is negligible. Neglecting the third term in $(\mathbb{B . 7})$ we can solve for $\dot{\phi}(T)$ in terms of $a(T)$ :

$$
\dot{\phi}(T) \approx \frac{c}{a(T)^{d-1}}
$$

where $c$ is a constant. Substituting (B.8) back into (B.5) (and ignoring $V$ and the 1, since they are both negligible near the singularity), we obtain a first order differential equation for $a(T): \dot{a}^{2}=\frac{c^{2}}{(d-1)(d-2)} \frac{1}{a^{2 d-4}}$. Taking the (negative branch) square root and integrating, we have

$$
a(T) \approx\left[c \sqrt{\frac{d-1}{d-2}}\left(T_{s}-T\right)\right]^{\frac{1}{d-1}}
$$

where $T_{s}$ corresponds to the time of the singularity, $a\left(T_{s}\right)=0$. Finally, using $(\overline{B .9})$ in (B.8) and integrating yields $\phi(T)$ :

$$
\phi(T) \approx \sqrt{\frac{d-2}{d-1}} \ln \left(T_{s}-T\right)+\text { const }
$$

This verifies that there is a singularity in all dimensions. 


\section{B.2. Black hole estimates}

Let us now turn to the second step, namely estimating the mass of the black hole required to cloak the singularity. This involves a bit more detailed analysis, since it depends on the behaviour of the null geodesics in the dynamical background. We will use the same procedure as before, and estimate at what time does the pure AdS metric cease to approximate the actual geometry. We will then use that as the cutoff for the ingoing radial null geodesic starting at $T=0$ at radius $R_{0}$; namely, we approximate the black hole size $r_{h 0}$ by the proper radius reached by this null geodesic at the cut-off time $T_{\text {trust }}, c f$. , Fig.4. In Appendix B.4 we will contrast these analytical estimates with numerical calculations in the FRW geometry.

Since we are starting with a time symmetric configuration at $T=0$, at early times $\dot{\phi}(T) \approx 0$, so that from (B.5) we have $\dot{a}^{2}-\frac{2 V}{(d-1)(d-2)} a^{2}=1$. Denoting $H^{2}=$ $-\frac{2}{(d-1)(d-2)} V\left(\phi_{0}\right)$ as before, we find the early time solution

$$
a(T) \approx \frac{\cos (H T)}{H}
$$

(Note that $H \gtrsim 1$, so that the singularity must occur at $T_{s} \lesssim \frac{\pi}{2}$. That is, for pure AdS, there is a coordinate singularity when $a$ vanishes at $T_{s}=\frac{\pi}{2 H}$; and whenever there is a nontrivial field, $a(T) \rightarrow 0$ even faster, as evident from (B.5). But in that case, the singularity is a genuine curvature singularity since $\phi \rightarrow \infty$ in addition to $a \rightarrow 0$.) From (B.5) we see that the scale factor starts deviating from this form when

$$
a^{2} \dot{\phi}^{2} \sim(d-1)(d-2)
$$

What is $\phi$ ? Initially, $\phi$ grows exponentially as it rolls down the quadratic potential, $\phi(T) \approx \phi_{0} \cosh \left(\frac{d-1}{2} T\right)$ where $\phi_{0}=\phi(0)$ is the initial value of the field in the internal region; but at relatively early times $T_{0} \approx \frac{1}{\sqrt{d-1}}$, when $V_{, \phi}<\frac{\dot{a}}{a} \dot{\phi}, \phi(T)$ changes form and starts behaving as in (B.8). Matching at $T_{0}$, we determine the constant $c \approx \eta \phi_{0}$, where $\eta \approx 0.44$ for $d=3$ and 1.41 for $d=5$. Substituting this back into (B.12), we see that the scale factor changes form when $a \approx[(d-1)(d-2)]^{-\frac{1}{2 d-4}} c^{\frac{1}{d-2}}$. Matching to the initial form of $a$, we find that the metric no longer approximates pure AdS at time $T_{\text {trust }}$, given by

$$
\varepsilon \equiv \frac{\pi}{2 H}-T_{\text {trust }}=v \phi_{0}^{\frac{1}{d-2}}
$$

For $d=3, v \approx 0.31$, whereas for $d=5, v \approx 0.74$. (Note that at this point, the field is still small: $\phi\left(T_{\text {trust }}\right) \approx \phi_{0}^{\frac{1}{d-1}} \ll 1$ for $\phi_{0} \ll 1$; so the quadratic approximation of the potential should be reasonable, and we can easily verify that at $T_{\text {trust }}, V \ll \dot{\phi}^{2}$.) 


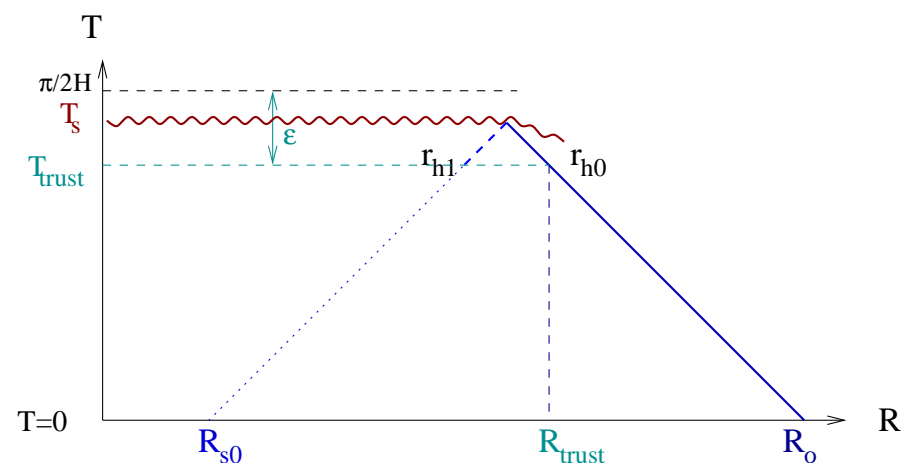

Fig. 4: "Causal" diagram in FRW coordinates (figure not to scale) for obtaining estimates of the horizon size.

We now estimate the black hole size by simply taking it to be the proper radius $r_{h 0} \approx r\left(T_{\text {trust }}\right)=R\left(T_{\text {trust }}\right) a\left(T_{\text {trust }}\right)$, determined by ray-tracing back the ingoing radial null geodesic starting from $R_{*}$ at $T=0$ (cf. Fig.4). Solving for the radial null geodesics in the geometry (B.1) with (B.11), we obtain 24

$$
R(T)=\frac{R_{*}\left(R_{*}+\sqrt{R_{*}^{2}+1}\right) \cos ^{2}(H T) \pm \sin (H T)}{\left(R_{*}+\sqrt{R_{*}^{2}+1}\right)(1+\sin (H T)) \cos (H T)}
$$

where the \pm denotes outgoing and ingoing geodesics, respectively. Note that $R(T)$ diverges when $\cos (H T)=0$; however, the proper radius $r(T)=R(T) \frac{\cos (H T)}{H}$ stays finite there. At $T=T_{\text {trust }}=\frac{\pi}{2 H}-\varepsilon$, the scale factor is simply $a\left(T_{\text {trust }}\right)=\frac{1}{H} \cos \left(\frac{\pi}{2}-\varepsilon H\right) \approx \varepsilon$ and (B.14) also simplifies. To the first subleading terms in $\varepsilon^{2}, \phi_{0}^{2}$, and $1 / R_{0}^{2}, R_{\text {trust }}=R\left(T_{\text {trust }}\right)$ becomes

$$
R_{\text {trust }} \approx \frac{-1+2 R_{0}^{2} \varepsilon^{2}\left(1+\frac{5}{8 R_{0}^{2}}+\frac{1}{2} \frac{d-1}{d-2} \phi_{0}^{2}\right)}{4 R_{0} \varepsilon}\left[1-\frac{1}{4 R_{0}^{2}}-\frac{1}{2} \frac{d-1}{d-2} \phi_{0}^{2}\right]
$$

Note that in order to maintain $R_{\text {trust }}$ being positive, so that the domain of dependence of the internal region includes the singularity, we must have $2 R_{0}^{2} \varepsilon^{2}>1$. Using (B.13), we must satisfy the following constraint on $\phi_{0}$ and $R_{0}$ :

$$
R_{0} \phi_{0}^{\frac{1}{d-2}}>\frac{1}{v \sqrt{2}} \sim 1
$$

24 This formula is valid even in the strong field regime as long as $T \leq T_{\text {trust. }}$. In the weak field regime $H \approx 1+\frac{1}{6} \phi_{0}^{2} \sim 1$, and so $R_{*} \sim R_{0}$. In the strong field regime we have to take into account the fact that proper radius of the spheres are shrunk by a factor of $H$; since $R_{0}$ is proper size of the homogeneous region in unit sized AdS geometry, we should take $R_{0}=H R_{*}$. 
Kepping all the coefficients in the above estimate, the RHS is 0.96 for $d=5$ and 2.28 for $d=3$. Without this condition, we are not guaranteed that the initial field configuration evolves to a singularity.

Note that [14 have a much more conservative procedure to estimate the horizon radius: They ray-trace the null geodesic from $R_{0}$ up to the singularity and then back to the initial data slice, to obtain $R_{s 0}$, as sketched in Fig.4. (Actually, to be able to ray-trace in pure AdS geometry, they bounce off from $T=T_{\text {trust }}$ rather than $T=T_{s}$.) The logic is that by the area theorem for black holes, a real horizon, which on the initial data slice must be larger than $R_{s 0}$ in order to cloak the singularity, could only grow in time. So $R_{s 0}$ gives a lower bound on the horizon radius and correspondingly the black hole mass $M_{\mathrm{BH}}$. To show that naked singularities must be produced, we want to show that the lower bound on $M_{\mathrm{BH}}$ is still larger than $M_{\text {config }}$, so we want to make this lower bound of $M_{\mathrm{BH}}$ as large as possible. As one can easily check, in AdS geometry, the proper radius $r(T)=a(T) R(T)$ grows along the outgoing geodesics. This means that it is profitable to estimate the horizon radius by the radius near 25 the singularity rather than at the initial data slice, i.e., by $r_{h 1}$ in Fig.4.

Note however that above, we estimate the black hole size by $r_{h 0}$ rather than $r_{h 1}(c f$. Fig.4). To check how much error we are making in estimating $r_{h 0}$ based on terminating ingoing null geodesic at $T=T_{\text {trust }}$, rather than continuing all the way to the singularity, we can estimate what the proper radius $r_{h 1}$ would be if we ray-traced the ingoing null geodesic from $r_{h 0}$ up to $T_{s}$ in the near-singularity geometry, bounced from the singularity, and raytraced backwards along the outgoing null geodesic back to $T_{\text {trust }}$. We then obtain $r_{h 1}=$ $a\left(R_{\text {trust }}\right) \sinh \left[\sinh ^{-1} R_{\text {trust }}-0.2\right]$, where the numerical factor 0.2 is only very weakly dependent on $d$. Thus, for $R_{\text {trust }}>\mathcal{O}(1)$, we can check that we are not making a very large error: $r_{h 1} \sim 0.82 r_{h 0}$.

Now, suppose $R_{0} \phi_{0}^{\frac{1}{d-2}} \gg 1$, so that the singularity forms in a large region, $R_{\text {trust }} \sim$ $\frac{1}{2} \varepsilon R_{0}$. Then the horizon radius which would cloak this singularity would have to be at least

$$
r_{h 0} \sim a\left(T_{\text {trust }}\right) R_{\text {trust }} \approx \frac{v^{2}}{2} R_{0} \phi_{0}^{\frac{2}{d-2}}
$$

In five dimensions we find $r_{h 0}=0.27 R_{0} \phi_{0}^{2 / 3}$. Note that since this corresponds to the

25 One has to be a bit careful, because in the near-singularity regime (B.9), $r(T) \rightarrow 0$ as $T \rightarrow T_{s}$ along outgoing radial geodesics which really terminate at the singularity in the domain of dependence of the inner region. 
proper radius, which includes the shrinking scale factor, this quantity may or may not be large, depending on $\phi_{0}$. In the text we quote $r_{h 0}$ in equation (3.4), as a lower bound on the size of the black hole.

We should point out that the order one coefficient in the above formula can be accurately determined numerically, by studying the FRW equations (B.5), (B.7), see Fig 6 . Our numerical studies in five dimensions shows that $r_{h 0}=0.37 R_{0} \phi_{0}^{2 / 3}$. The correction comes from the inaccuracy in our ability to accurately pin down the matching point $T_{\text {trust }}$ and the time to the singularity $T_{s}$ (we have been using the upper bound $\frac{\pi}{2 H}$ ).

The mass of the Schw-AdS $S_{d}$ black hole is given by $M_{\mathrm{BH}}=(d-2) \omega_{d} r_{h 0}^{d-3}\left(r_{h 0}^{2}+1\right)$ where $\left.\omega_{d} \equiv \pi^{\frac{d-1}{2}} / \Gamma\left(\frac{d-1}{2}\right)\right)$. If $r_{h 0} \gg 1$, then we can estimate the mass of the large AdS black hole by

$$
M_{\mathrm{BH}}=\omega_{d} \varsigma R_{0}^{d-1} \phi_{0}^{\frac{2 d-2}{d-2}}
$$

where $\varsigma \approx 0.0023$ for $d=3$ and $\varsigma \approx 0.017$ for $d=5$.

For the configuration mass given approximately by $M_{\text {config }} \sim \omega_{d} R_{0}^{d-1} \phi_{0}^{2}$, we have (in the large black hole approximation)

$$
\mu \sim \varsigma \phi_{0}^{\frac{2}{d-2}} \ll 1 .
$$

This means that we are kinematically likely to produce black holes, i.e., the energy of the starting configuration greatly exceeds that of the requisite black hole. As we see, $\mu$ gets larger (and therefore slightly more favorable to produce naked singularities) for larger $\phi_{0}$ and in higher dimensions; but since $\varsigma \ll 1$, unfortunately there is no numerical window of opportunity wherein we could have $\phi_{0}<1$ while $\mu>1$. In (B.19), $\mu>1$ would require $\phi_{0}>\mathcal{O}(100)$ which is well outside the linearized regime where (B.19) was valid.

\section{B.3. FRW cosmology with strong fields}

In Appendix B.1. we have modeled the dynamics in the domain of dependence of the homogeneous central region of all of our configurations in terms of FRW cosmology with hyperbolic spatial sections. The details of the dynamics depend on the dimension and on the precise form of the scalar potential $V(\phi)$. We are interested in the asymptotic large $\phi$ behaviour for the exponential supergravity potential (2.3). For $\phi \gg 1$ we can ignore the second term in (2.3) and so we will consider a generic negative definite exponential potential $V(\phi)=-v \exp (2 k \phi)$. The equations governing the dynamics are any two of (B.5), (B.6), (B.7), and we have time symmetric boundary conditions along with $\phi(0)=\phi_{0} \gg 1$. 
Introduce a new field $\psi=\phi-\phi_{0}$. In terms of $\psi$ we see that $V(\psi)=$ $-v e^{2 k \phi_{0}} \exp (2 k \psi)$. Since the potential rescales with the field redefinition, it is tempting to scale the factor $e^{k \phi_{0}}$ out of the problem. In fact, by rescaling the time coordinate $T=e^{-k \phi_{0}} U$ and the scale factor $b(U)=e^{k \phi_{0}} a(U)$ we obtain the equations:

$$
\begin{gathered}
\dot{b}^{2}-\frac{2}{(d-1)(d-2)} b^{2}\left(\frac{1}{2} \dot{\psi}^{2}-v e^{2 k \psi}\right)=1 \\
\ddot{b}+\frac{2}{(d-1)(d-2)}\left(v e^{2 k \psi}+\frac{(d-2)}{2} \dot{\psi}^{2}\right)=0 \\
\ddot{\psi}+(d-1) \frac{\dot{b}}{b} \dot{\psi}-2 v k e^{2 k \psi}=0
\end{gathered}
$$

We use the overdot to denote derivatives with respect to $U,{ }^{\cdot} \equiv \frac{d}{d U}$. This rescaling has the effect that the scale $e^{k \phi_{0}}$ drops out of the dynamics. In the resulting rescaled problem the time to singularity will be determined solely by the number $\tilde{H}^{2}=\frac{2 v}{(d-1)(d-2)}$. The equations (B.20), (B.21), (B.22), will collapse to a singularity $b(U) \rightarrow 0$ in a finite time $U_{s}$, which is a finite fraction of the rescaled AdS time $\pi / 2 \tilde{H}$. Hence we see that $T_{s} e^{k \phi_{0}}$ is finite; the homogeneous region exists for a finite fraction of the original AdS time $\pi / 2 H=$ $\pi e^{k \phi_{0}} / 2 \tilde{H}$ before the big crunch singularity.

\section{B.4. Numerical estimates of black hole size}

In Appendix B.2 we discussed the size of the black hole required to cloak the singularity. Most of our estimates there were based on $\phi_{0} \ll 1$, and we derived the formula $r_{h 0} \sim R_{0} \phi_{0}^{2 / 3}$ in this regime. We will improve on this below. It is clear that as we increase $\phi_{0}$ the singularity will happen on much shorter timescales, since the backreaction on the geometry is going to be more important. One might naively think that in the regime $\phi_{0} \gg 1$ the singularity will encompass all of the homogeneous region and in particular estimate $r_{h 0} \sim R_{0}$.

To get a reliable estimate on the size of the black hole necessary to cloak the singularity, we solve FRW equations (B.5), (B.6), and (B.7) numerically. With an explicit numerical solution to the scale factor $a(T)$, we can study the property of null geodesics in the resulting big crunch geometry. 

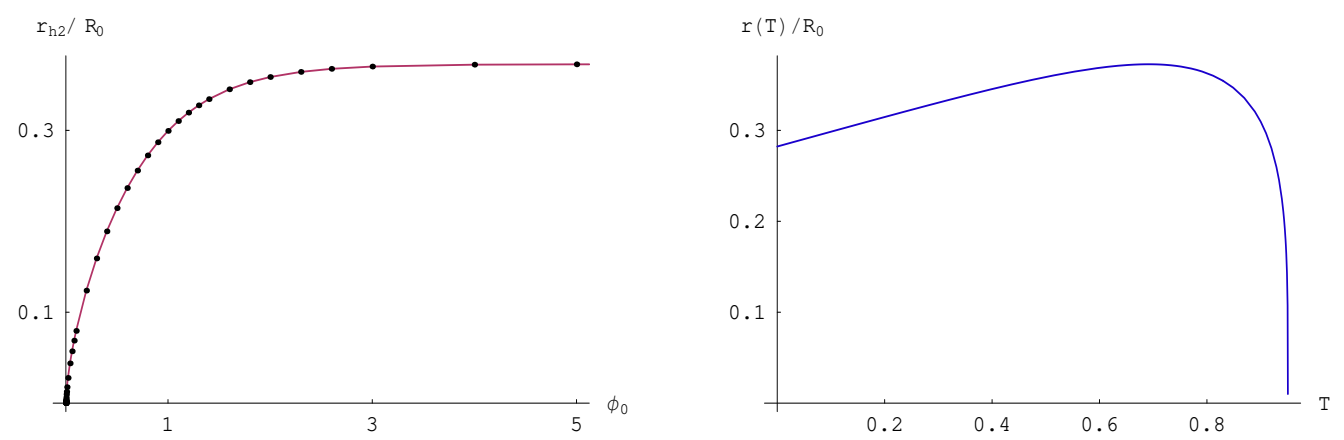

Fig. 5: Numerical estimates for $r_{h 2}\left(\phi_{0}\right)$. (a) $r_{h 2}\left(\phi_{0}\right) / R_{0}$ evaluated from integrating the geodesic equations. (b) Proper radius as a function of time in the rescaled geometry. The maximum value is attained at $T=T_{\max }$.

In particular we can obtain the behavior of $r_{h 0}\left(\phi_{0}\right)$. As indicated in Fig.4, we start from a point on the initial data slice where the spheres have proper size $R_{0}$ and consider a radially ingoing null geodesic. At some point very close to the singularity we cut-off the geometry and from the end-point of the geodesic from $R_{0}$ onto this cut-off surface we take a radially ingoing null geodesic. This would intersect the initial data slice at $R_{s 0}$. However, the proper size of the spheres will peak at some intermediate time $T_{\max }$. Call this radius $r_{h 2}$; this will be the lower bound on the size of the black hole. We use the numerical solution for $a(T)$ to estimate $r_{h 2}$. In Fig.5 we show the behavior of $r_{h 2}\left(\phi_{0}\right) / R_{0}$. We see that for large $\phi_{0}, r_{h 2}$ is independent of $\phi_{0}$ as expected. Moreover, we find that for small $\phi_{0}$,

$$
r_{h 2}\left(\phi_{0}\right)=0.37 R_{0} \phi_{0}^{2 / 3}
$$

As mentioned in Appendix B.2, the numerical evaluation of $r_{h 2}$ differs from our weak field estimate $r_{h 0}$ only in the numerical factor, which is attributable to a more accurate determination of the point where the geometry deviates significantly from pure AdS.

An extremely efficient way to estimate the asymptotic value of $r_{h 2}\left(\phi_{0}\right)$ as $\phi_{0} \rightarrow \infty$ is to use the scaling argument of Appendix B.3. We basically just have to solve the rescaled equations, say ( $(\overline{\mathrm{B} .20})$, and $(\overline{\mathrm{B} .22})$ to find $b(U)$. Armed with the knowledge of $b(U)$ we can trace the null geodesics and see where the maximum occurs (see Fig.5). We find

$$
r_{h 2} \rightarrow 0.372 R_{0}, \quad \text { for } \quad \phi_{0} \gg 1
$$

\section{B.5. Refining the estimate of the black hole size}

The estimates for the black hole size (B.23), (B.24), do not constitute the tightest lower bound. In deriving them we assumed that the singularity stretched out to the boundary 


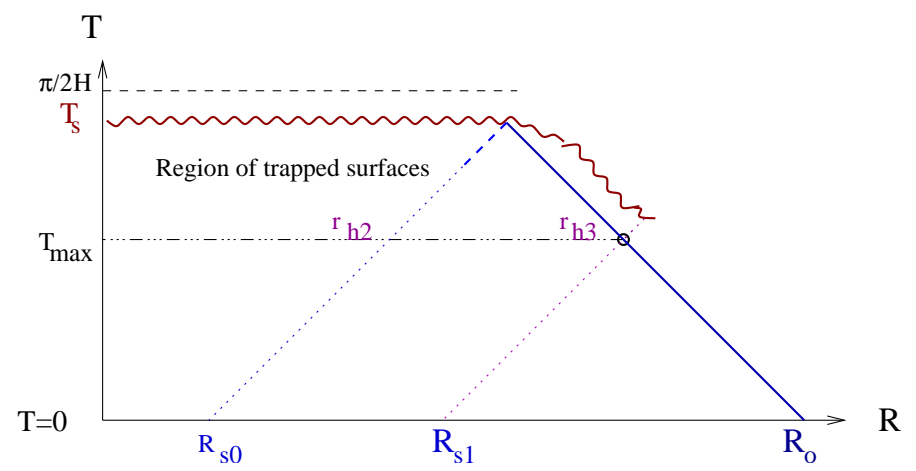

Fig. 6: "Causal" diagram in FRW coordinates (figure not to scale) with the new information to refine the size of the singularity.

of the domain of dependence at time $T_{s}$. However, we now argue that it necessarily has to be bigger and so obtain more stringent bounds.

In estimating $r_{h 2}$ we used the essential fact that the congruence of outgoing null geodesics starting from a point $R_{s 0}$ on the initial data surface first expands and then contracts in size, attaining a maximum at $T_{\max }$. This implies that the surface of constant FRW time, $T=T_{\max }$, is a marginally trapped surface. Since the region $T>T_{\max }$ is a region of trapped surfaces, any geodesic entering it must necessarily end at the singularity.

Hitherto, we picked the congruence starting from $R_{s 0}$ that meets up at the singularity with the ingoing null congruence emanating from $R_{0}$. Now consider an outgoing null congruence that starts at $R_{s 0}+\delta R$. This congruence will initially start out expanding. Can it expand for ever and reach the boundary of AdS? We will argue that for sufficiently small $\delta R$ it can not. First of all, the geodesics will have to enter the region of trapped surfaces $T>T_{\max }$, and hence will start contracting in this region. Moreover, it is also clear that this congruence will exit the domain of dependence of the homogeneous central region before meeting with the singularity, see Fig.6. If these geodesics were to miss the singularity, then they will end up at the AdS boundary. However, this will be a clear violation of the Raychaudhuri equation for geodesic congruences, which implies that a contracting congruence of null geodesics cannot re-expand, so long as the matter contributing to the stress tensor satisfies the null energy condition 26. For the congruence in question, there is only one possible outcome - it must end up at the singularity once it enters the region of

26 For the scalar-gravity action (2.2), the stress-tensor is given before (B.4). Despite the negative-definite potential, the null energy condition, $T_{\mu \nu} k^{\mu} k^{\nu} \geq 0 \forall$ null $k^{\mu}$ is satisfied, since the contraction with a null vector projects out the potential contribution. 

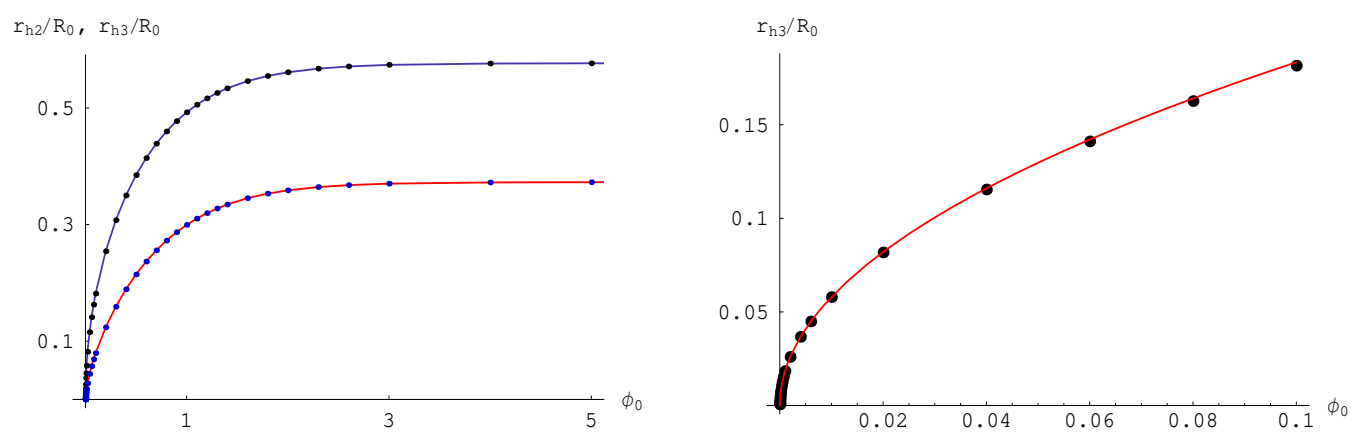

Fig. 7: Numerical estimates for $r_{h 3}\left(\phi_{0}\right)$.

(a) Comparing $r_{h 3}\left(\phi_{0}\right) / R_{0}$ (upper curve) to $r_{h 2}\left(\phi_{0}\right) / R_{0}$ (lower curve).

(b) Weak field estimate of $r_{h 3}\left(\phi_{0}\right)$ and comparison to the analytic fit (B.25).

trapped surfaces. This in particular implies that the singularity must extend outside the domain of dependence of the initial data surface. A similar argument was used by [14] to argue that the singularity should have a finite proper size.

Having established that the singularity must stretch outside the domain of dependence of the region $r \leq R_{0}$, we would like to obtain the best estimate for $\delta R$. On the constant FRW time $T=T_{\max }$ surface, the proper size of the geodesics is a monotonically increasing function of $\delta R$. This implies that the best estimate for the proper size is achieved by moving out to the boundary of the domain of dependence. So we only need to know the proper size of the spheres at the boundary of the domain of dependence (and hence by definition on the ingoing congruence from $R_{0}$ ) at $T_{\max }$. From Fig.7, we see that the outgoing null congruence from $R_{s 1}$ is the one where the maximum proper size $r_{h 3}$ is reached at the boundary. For larger starting $R$, there is no maximal size sphere along an outgoing congruence within the domain of dependence of the homogeneous region, and we would have to resort to full numerical simulation to make further refinements to our estimate.

Using the numerical solution for the scale factor one can ascertain $r_{h 3}$. We show in Fig.7 the numerical estimates for the same and compare it also to the previously derived results of Appendix B.4. We find that in the weak field limit we have

$$
r_{h 3}\left(\phi_{0}\right)=0.58 R_{0} \phi_{0}^{1 / 2}, \quad \text { for } \quad \phi_{0} \ll 1
$$

This is parametrically larger than our previous estimate (B.23) by a factor of $\phi_{0}^{-1 / 6}$. In the strong field limit we obtain

$$
r_{h 3} \rightarrow \sigma_{s} R_{0}=0.577 R_{0}, \quad \text { for } \quad \phi_{0} \gg 1
$$


This betters our previous estimate (B.24) by about $55 \%$, which is a decent improvement, but sadly insufficient to rigorously rule out black hole formation. It is likely that the singularity stretches out a bit more than what we estimated above, but this is not something we can estimate reliably within the domain of dependence of the homogeneous region i.e., in the FRW cosmology. We therefore make our estimates for black hole masses using the size of the horizon to be given by $r_{h 3}$. The values of $r_{h 3}$ are quoted as the lower bound for $R_{s}$ in the text in (3.8) and (3.10), for weak and strong fields, respectively.

These results can easily be generalized to other dimensions. In order to obtain the strong field behaviour of $r_{h 3}$, we need to know the exact form of the scalar potential. In the weak field limit we can make progress using the quadratic approximation to the scalar potential. For configurations analogous to (2.4), the mass can be determined from (A.20) to be $M_{\text {config }}=\frac{(d-1)}{4} \omega_{d} \phi_{0}^{2} R_{0}^{d-1}$. The black hole mass, with horizon size $r_{h 3}$ is of course, $M_{B H}=(d-2) \omega_{d} r_{h 3}^{d-3}\left(r_{h 3}^{2}+1\right)$. In $d=7$, with $\phi_{0}=0.01$ we find $r_{h 3}=0.148 R_{0}$, leading to the parameter $\mu$, as defined in (3.6), being $\mu=0.35$. For smaller $\phi_{0}$, say $\phi_{0}=0.001$, we find $r_{h 3}=0.0693 R_{0}$ implying $\mu=0.37$. We see therefore that $\mu$ gets a little bigger as the number of dimensions increases, but far too slowly to make the case for singularity formation in higher dimensions.

\section{B.6. The double well potential scenario}

Let us now return to the double well potential scenario discussed in [7]. As mentioned in the text, there is a gap in the arguments for violating cosmic censorship, which we now briefly discuss. The minimizing field configuration, which is guaranteed to have energy $\sim R_{0}$, does not have exactly constant profile in the interior region. As suggested by the numerical analysis of [17] and later explained by [16], this leads to the actual size of the singularity being smaller than assumed in [7], and in fact the black hole required to cloak this smaller singularity may require less mass than that available in the configuration.

Conversely, suppose we keep the field profile homogeneous in the interior region. One might hope this does not increase the mass too much, whereas the size of the singularity is as given by the original estimates. If that were the case, then as long as one started with sufficiently large $R_{0}$, one could retain the kinematic arguments for forming naked singularities. Unfortunately, the mass does increase too much, a point made independently in [16]. Here we will demonstrate this statement numerically, since we can use essentially the same analysis as above to bound the size of the singularity and thereby ascertain whether or not the requisite black hole mass is sufficiently large. 
For small fields, we can use the quadratic approximation of the potential. Consider therefore the dynamics of the scalar field in the potential given by $V(\phi)=-3+\frac{1}{2} m^{2} \phi^{2}$. This generalizes the situation considered by [7] by introducing an arbitrary mass parameter $m$. While the field typically oscillates in the potential with the frequency given by $m$ and then diverges logarithmically, the scale factor vanishes in very similar manner as before. Repeating the numerical analysis of the preceding section, we obtain (cf. Fig.6) $r_{h 2} \sim \phi_{0}$ while $r_{h 3} \approx c \phi_{0}^{2 / 3}$, with $c \sim 0.2 m^{4 / 3}$ for small $m$. Even for larger $m$ the coefficient remains small; for example, for $m^{2}=100, c \approx 0.3$. Using the estimate for the mass of the configuration, $M_{\text {config }} \sim m^{2} \phi_{0}^{2} R_{0}^{3}$, we can easily calculate the parameter $\mu$.

\begin{tabular}{|c|rr|}
\hline$\mu$ & $\phi_{0}=0.0001$ & $\phi_{0}=0.1$ \\
\hline$m^{2}=100$ & 0.00015 & 0.05 \\
$m^{2}=1$ & 0.024 & 0.028 \\
$m^{2}=0.01$ & 0.00037 & 0.00037 \\
\hline
\end{tabular}

Table 1: Values of $\mu$ for a few representative values of $m^{2}$ and $\phi_{0}$.

Several representative values of $\mu$ are tabulated in Table 1 . We see that none of these cases yields $\mu>1$, needed for the kinematic argument for forming naked singularities. The table suggests that the most promising region to consider is that of large masses and large fields 227. However, closer exploration of this region did not yield any parameters giving rise to $\mu>1$.

\section{Appendix C. Probe brane potentials}

We are interested in the supergravity dual to $\mathcal{N}=4 \mathrm{SYM}$ deformed by the operator $\mathcal{O}_{2}$. Our strategy will be to start with five dimensional gauged supergravity and lift the solution to ten dimensional Type IIB supergravity. By the standard AdS/CFT dictionary, we need to find a solution to the scalar-gravity system (2.2), with the scalar filed profile asymptotically behaving as the non-normalizable mode.

The deformation by $\mathcal{O}_{2}$ will result in a static, spherically symmetric configuration. So we can use a metric ansatz identical to that of the scalar hair black hole (A.21) in

27 The value of $\phi_{0}$ is bounded from above by the requirement that $V\left(\phi_{0}\right)<0$, which effectively restricts us to the small field regime for large $m^{2}$. 
Appendix A, i.e.,

$$
d s_{5}^{2}=-e^{-2 \delta(r)} f(r) d t^{2}+\frac{1}{f(r)} d r^{2}+r^{2} d \Omega_{3}^{2}
$$

We will also have a scalar field profile $\phi(r)$. The equations of motion are as in (A.22).

Given this solution it is simple to write down the full 10-dimensional geometry using the consistent truncation ansatz. Let $\xi$ be a coordinate on the $\mathbf{S}^{5}$, so that the round $\mathbf{S}^{5}$ metric takes the form:

$$
d \Omega_{5}^{2}=d \xi^{2}+\sin ^{2} \xi d \psi^{2}+\cos ^{2} \xi d \Omega_{3}^{2} .
$$

The full 10-d solution is read off from [21] to be

$$
d s_{10}^{2}=\Delta d s_{5}^{2}+F \Delta d \xi^{2}+\frac{F^{2}}{\Delta} \sin ^{2} \xi d \psi^{2}+\frac{1}{F \Delta} \cos ^{2} \xi d \Omega_{3}^{2}
$$

where

$$
\Delta^{2}=\frac{1}{F^{2}} \sin ^{2} \xi+F \cos ^{2} \xi, \quad F=\exp \left(\frac{1}{2 \sqrt{3}} \phi\right)
$$

The five-form is given as

$$
G_{5}=-U \epsilon_{5}-3 \sin \xi \cos \xi \frac{1}{F} * d F \wedge d \xi,
$$

with

$$
U=-2\left(F^{2} \cos ^{2} \xi+\frac{1}{F}\left(1+\sin ^{2} \xi\right)\right) .
$$

The $\epsilon_{5}$ and the Hodge dual are with respect to the five dimensional metric $d s_{5}^{2}$. In particular,

$$
\epsilon_{5}=-r^{3} e^{-\delta} d t \wedge d r \wedge d \Omega_{3} .
$$

The brane probe action is composed of the DBI term and the WZ term as:

$$
S_{D 3}=-\tau_{3} \int d^{4} \zeta \sqrt{\operatorname{det} G^{(i n d)}}+\mu_{3} \int C_{4}
$$

We use static gauge $\zeta^{0}=t, \zeta^{i}=\Omega_{3}^{i}$ and evaluate

$$
\sqrt{\operatorname{det} G^{(i n d)}}=r^{3} \sqrt{f} \Delta^{2} e^{-\delta}
$$

To evaluate the four form $C_{4}=C(r, \xi) d t \wedge d \Omega_{3}$ we use

$$
\frac{\partial C(r, \xi)}{\partial r}=-U r^{3} e^{-\delta}
$$


To find the exact probe potential we need to know the precise form of the functions $F(r), f(r)$ and $\delta(r)$. This can be done numerically, but it is relatively simple to extract the asymptotic form of the potential for large $r$. To this end, realize that our asymptotic boundary conditions are:

$$
\begin{aligned}
& f(r) \sim 1+r^{2} \\
& \phi(r) \sim \alpha \frac{\log (r)}{r^{2}} \\
& \delta(r) \sim \frac{1}{3} \alpha^{2} \frac{\log (r)^{2}}{r^{4}}
\end{aligned}
$$

The behaviour of $\delta(r)$ is determined by its equation of motion (A.22). This will not be essential in what follows as its effect is at a subleading order.

With these ingredients in hand, one simply expands out the contributions from the DBI term (C.9) and the WZ term (which is just $C(r, \xi)$ ) to the potential. We have

$$
\begin{gathered}
r^{3} \sqrt{f} \Delta^{2} e^{-\delta} \sim r^{4}+\frac{\alpha}{\sqrt{3}} r^{2} \log (r)\left(\frac{1}{2} \cos ^{2} \xi-\sin ^{2} \xi\right)+\frac{1}{2} r^{2}+\cdots \\
C(r, \xi)=r^{4}+\frac{\alpha}{\sqrt{3}}\left(r^{2} \log (r)-\frac{1}{2} r^{2}\right)\left(\cos ^{2} \xi-\frac{1}{2}\left(\sin ^{2} \xi+1\right)\right)+\cdots
\end{gathered}
$$

Putting both of these together and using the fact that $\mu_{3}=\tau_{3}$ for a BPS D3-brane, we see that the leading order terms cancel among the two contributions. This is guaranteed by the supersymmetries preserved by the AdS background, since at this order we are not picking up any contributions from the deformation $\phi(r)$. As a result we we are left with an effective potential:

$$
V_{e f f}=\frac{\tau_{3}}{2} r^{2}\left(1-\frac{\alpha}{2 \sqrt{3}}\left(3 \sin ^{2} \xi-1\right)\right)
$$

We see that for $\alpha>\alpha_{c}=2 \sqrt{3}$, the potential is unbounded from below. A similar computation done in the case of the Poincare patch of AdS by [27] reveals that the critical value of $\alpha$ for the field theory on $\mathbf{R}^{4}$ is zero, i.e., the field theory is always unstable under the deformation. Note that the salubrious term $\frac{\tau_{3}}{2} r^{2}$ arises here because of the curvature of the $\mathbf{S}^{3}$ and can be traced to the constant in $f(r)=1+r^{2}$. 


\section{References}

[1] S. W. Hawking and G. F .R. Ellis, The Large Scale Structure of Space-Time, Cambridge University Press, (1973).

[2] R. Penrose, Gravitational Collapse: The Role Of General Relativity, Riv. Nuovo Cim. 1, 252 (1969) [Gen. Rel. Grav. 34, 1141 (2002)].

[3] R. M. Wald, Gravitational collapse and cosmic censorship, arXiv:gr-qc/9710068].

[4] M. W. Choptuik, Universality And Scaling In Gravitational Collapse Of A Massless Scalar Field, Phys. Rev. Lett. 70, 9 (1993).

[5] P. S. Joshi and I. H. Dwivedi, Naked Singularities In Spherically Symmetric Inhomogeneous Tolman-Bondi Dust Cloud Collapse, Phys. Rev. D 47, 5357 (1993) arXiv:grqc/9303037.

[6] V. E. Hubeny, Overcharging a Black Hole and Cosmic Censorship, Phys. Rev. D 59, 064013 (1999) arXiv:gr-qc/9808043.

[7] T. Hertog, G. T. Horowitz and K. Maeda, Generic cosmic censorship violation in anti de Sitter space, [arXiv:gr-qc/0307102].

[8] K. Maeda, T. Torii and M. Narita, Do naked singularities generically occur in generalized theories of gravity?, Phys. Rev. Lett. 81, 5270 (1998) arXiv:gr-qc/9810081.

[9] J. Maldacena, The Large N Limit of Superconformal Field Theories and Supergravity, Adv. Theor. Math. Phys. 2 (1998) 231, arXiv:hep-th/9711200].

[10] O. Aharony, S.S. Gubser, J. Maldacena, H. Ooguri, Y. Oz, Large N Field Theories, String Theory and Gravity, Phys. Rept. 323 (2000) 183, arXiv:hep-th/9905111.

[11] E. Witten, Anti De Sitter Space And Holography, Adv. Theor. Math. Phys. 2 (1998) 253, arXiv:hep-th/9802150.

[12] S. Gubser, I. Klebanov, and A. Polyakov, Gauge Theory Correlators from Non-Critical String Theory, Phys. Lett. B428 (1998) 105, arXiv:hep-th/9802109.

[13] L. Fidkowski, V. Hubeny, M. Kleban and S. Shenker, The black hole singularity in $A d S / C F T$, arXiv:hep-th/0306170.

[14] T. Hertog, G. T. Horowitz and K. Maeda, Negative energy in string theory and cosmic censorship violation, arXiv:hep-th/0310054.

[15] M. Gutperle and P. Kraus, Numerical Study of Cosmic Censorship in String Theory, arXiv:hep-th/0402109

[16] T. Hertog, G. T. Horowitz and K. Maeda, Update on Cosmic Censorship Violation in $A d S$, arXiv:gr-qc/0405050].

[17] D. Garfinkle, Numerical simulation of a possible counterexample to cosmic censorship, arXiv:gr-qc/0403078.

[18] M. Alcubierre, J. A. Gonzalez, M. Salgado and D. Sudarsky, The cosmic censor conjecture: Is it generically violated?, arXiv:gr-qc/0402045. 
[19] P. Breitenlohner and D. Z. Freedman, Stability In Gauged Extended Supergravity, Annals Phys. 144, 249 (1982); Positive Energy In Anti-De Sitter Backgrounds And Gauged Extended Supergravity, Phys. Lett. B 115, 197 (1982).

[20] L. Mezincescu and P. K. Townsend, Stability At A Local Maximum In Higher Dimensional Anti-De Sitter Space And Applications To Supergravity, Annals Phys. 160, 406 (1985).

[21] M. Cvetic, H. Lu, C. N. Pope, A. Sadrzadeh and T. A. Tran, Consistent SO(6) reduction of type IIB supergravity on S(5), Nucl. Phys. B 586, 275 (2000) arXiv:hepth/0003103].

[22] H. J. Kim, L. J. Romans and P. van Nieuwenhuizen, The Mass Spectrum Of Chiral $N=2 D=10$ Supergravity On $S^{* *} 5$, Phys. Rev. D 32, 389 (1985).

[23] M. Dafermos, A note on naked singularities and the collapse of self-gravitating Higgs fields, arXiv:gr-qc/0403033.

[24] G. T. Horowitz and V. E. Hubeny, Quasinormal modes of AdS black holes and the approach to thermal equilibrium, Phys. Rev. D 62, 024027 (2000) arXiv:hepth/9909056].

[25] A. Frolov, in preparation.

[26] T. Torii, K. Maeda and M. Narita, Scalar hair on the black hole in asymptotically anti-de Sitter spacetime, Phys. Rev. D 64, 044007 (2001).

[27] J. Babington, D. E. Crooks and N. Evans, A non-supersymmetric deformation of the AdS/CFT correspondence, JHEP 0302, 024 (2003) arXiv:hep-th/0207076.

[28] N. Seiberg and E. Witten, The D1/D5 system and singular CFT, JHEP 9904, 017 (1999) arXiv:hep-th/9903224.

[29] D. Z. Freedman, S. S. Gubser, K. Pilch and N. P. Warner, Continuous distributions of D3-branes and gauged supergravity, JHEP 0007, 038 (2000) arXiv:hep-th/9906194.

[30] J. Polchinski and M. J. Strassler, "The string dual of a confining four-dimensional gauge theory," arXiv:hep-th/0003136.

[31] O. DeWolfe, D. Z. Freedman, S. S. Gubser, G. T. Horowitz and I. Mitra, Stability of $A d S(p) x M(q)$ compactifications without supersymmetry, Phys. Rev. D 65, 064033 (2002) arXiv:hep-th/0105047.

[32] J. M. Maldacena and A. Strominger, AdS(3) black holes and a stringy exclusion principle, JHEP 9812, 005 (1998) arXiv:hep-th/9804085.

[33] S. Deger, A. Kaya, E. Sezgin and P. Sundell, Spectrum of $D=6, N=4 b$ supergravity on $\operatorname{AdS(3)} x S(3)$, Nucl. Phys. B 536, 110 (1998) arXiv:hep-th/9804166.

[34] R. Dijkgraaf, Instanton strings and hyperKaehler geometry, Nucl. Phys. B 543, 545 (1999) arXiv:hep-th/9810210.

[35] A. Dhar, G. Mandal, S. R. Wadia and K. P. Yogendran, D1/D5 system with B-field, noncommutative geometry and the CFT of the Higgs branch, Nucl. Phys. B 575, 177 (2000) arXiv:hep-th/9910194. 
[36] J. Maldacena and L. Maoz, Wormholes in AdS, arXiv:hep-th/0401024.

[37] H. Nicolai and H. Samtleben, Kaluza-Klein supergravity on AdS(3) $x$ S(3), JHEP 0309, 036 (2003) arXiv:hep-th/0306202.

[38] L. Susskind, "Some Speculations About Black Hole Entropy In String Theory," arXiv:hep-th/9309145.

[39] G. T. Horowitz and J. Polchinski, "A correspondence principle for black holes and strings," Phys. Rev. D 55, 6189 (1997) arXiv:hep-th/9612146.

[40] C. V. Johnson, A. W. Peet and J. Polchinski, Gauge theory and the excision of repulson singularities, Phys. Rev. D 61, 086001 (2000) arXiv:hep-th/9911161.

[41] T. Damour and M. Henneaux, "Chaos in superstring cosmology," Phys. Rev. Lett. 85, 920 (2000) arXiv:hep-th/0003139. 